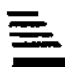

\section{AIAA-93-0535 \\ Preconditioning for the Navier-Stokes Equations with Finite-Rate Chemistry}
A.G. Godfrey
R.W. Walters
Virginia Polytechnic Inst. and State Univ. Blacksburg, VA
B. van Leer
University of Michigan Ann Arbor, MI

\section{1st Aerospace Sciences Meeting \& Exhibit January 11-14, 1993 / Reno, NV}

For permission to copy or republish, contact the American Institute of Aeronautics and Astronautics 370 L'Enfant Promenade, S.W. Washington, D.C. 20024 


\title{
PRECONDITIONING FOR THE NAVIER-STOKES EQUATIONS WITH FINITE-RATE CHEMISTRY
}

\author{
Andrew G. Godfrey ${ }^{\dagger}$ \\ Robert W. Walters $\ddagger$ \\ Department of Aerospace and Ocean Engineering \\ Virginia Polytechnic Institute and State University \\ Blacksburg, Virginia 24061 \\ Bram van Leer ${ }^{\ddagger}$ \\ Department of Aerospace Engineering \\ University of Michigan \\ Ann Arbor, Michigan $\$ 8109$
}

The preconditioning procedure for generalized finite-rate chemlstry and the proper preconditioning for the one-dimensional Navier-Stokes equations are presented. Elgenvalue stifiness is resolved and convergence-rate acceleration is demonstrated over the entire Mach-number range from the incompressible to the hypersonic. Specific benefits are reallzed at low and transonic flow speeds. The extended preconditioning matrix accounts for thermal and chemical non-equilibrium and its implementation is explained for both explicit and Implicit time marching. The effect of higher-order spatial accuracy and various flux spllttings is investigated. Numerical analysis reveals the possible theoretical improvements from using preconditioning at all Mach numbers. Numerical results confirm the expectations from the numerical analysls. Representative test cases include flows with previously troublesome embedded high-condition-number reglons.

\section{Nomenclature}

Sound speed

$A, B \quad$ Inviscid flux Jacobians

$A_{v} \quad$ Viscous flux Jacobian

$c_{v} \quad$ Specific heat at constant volume

$e \quad$ Mixture internal energy

$e_{0} \quad$ Mixture internal stagnation energy

$e_{n} \quad$ Mixture non-equilibrium energy

$f, g, h \quad$ Inviscid flux vectors

$h_{0} \quad$ Mixture internal stagnation enthalpy

$\hat{f} \quad$ Generalized-coordinate flux vector

$G \quad$ Gain matrix

$\vec{k}_{q}, \vec{l}_{q}, \vec{m}_{q} \quad$ Unit vectors

$L$

$m$

$\bar{M}$

$n, N$

$p$

$P$

$\mathcal{P}$
Characteristic length

Wave number

Mach number

Iteration time levels

Thermodynamic pressure

Preconditioning matrix for symmetrization variables Preconditioning matrix for numerical implementation

\footnotetext{
$\dagger^{\dagger}$ Research Assistant, Member AIAA
}

$\ddagger$ Professor, Fellow AIAA $\begin{array}{ll}\text { p } & 3 \times 3 \text { velocity submatrix in } \mathcal{P} \\ P_{T} & \text { Prandtl number }\end{array}$

$q \quad$ Primitive variables, velocity magnitude

$Q \quad$ Conservative variables

$R \quad$ Flux balance residual

Re Reynolds number

$t \quad$ Time

$T \quad$ Temperature

$u, v, w \quad$ Cartesian velocity components

$\tilde{u} \quad$ Velocity normal to cell face

$U$ Symmetrization variables aligned coordinate system

Vol Cell volume

$x, y, z \quad$ Cartesian coordinates

$Z$ Viscous symmetrization variables

$\alpha \quad$ Angle of attack

$\beta_{i-} \quad$ Defined thermodynamic variable

$\beta_{x} \quad$ Fourier frequency

$\gamma \quad$ Ratio of specific heats

$\epsilon^{n} \quad$ Error in solution at time level $n$

$\theta \quad$ Cell face normal angle

$\theta_{\bar{z}}, \theta_{\bar{y}}, \theta_{z}$ Direction cosine angles

$\lambda \quad$ Courant number, wave length, and eigenvalue

$\nu \quad$ Courant number, kinematic viscosity

$\xi, \eta, \zeta \quad$ Generalized and stream-aligned coordinates

II $3 \times 3$ velocity submatrix in $\mathcal{P}^{-1}$ 


$\begin{array}{ll}\rho & \text { Mixture density } \\ \rho_{i} & \text { Species density } \\ \rho_{s} & \text { Spectral radius of the gain } \\ \tau, \beta & \text { Preconditioning factors dependent } \\ & \text { upon Mach number }\end{array}$

Subscripts

$\begin{array}{ll}(\cdot)_{i} & \text { Species value } \\ L & \text { Left state variable } \\ R & \text { Right state variable } \\ x & \text { Component in the } x \text { direction } \\ y & \text { Component in the } y \text { direction } \\ z & \text { Component in the } z \text { direction } \\ 0 & \text { Stagnation quantity }\end{array}$

Accents

Unit vector or Generalized coordinate flux vector

Roe averaged quantity

Stream aligned quantity

Operators

$\begin{array}{ll}\Delta(\cdot) & \text { Forward difference operator } \\ {[\cdot]} & (\cdot)_{R}-(\cdot)_{L}\end{array}$

\section{Introduction}

The conservation equations for moderate and high Mach-number flows are well coupled, and standard numerical techniques perform adequately. However, in regions of low Mach-number flow, the energy and momentum equations decouple, and the fluid dynamics become stiff. The condition number quantifies the degree of stiffness and is the ratio of the largest to the smallest characteristic speed. When the smallest eigenvalue approaches zero (i.e. at slow, essentially incompressible flow and at transonic flow speeds), the condition number becomes prohibitively large. Characteristic time stepping or preconditioning eradicates this difficulty and allows us to simulate incompressible flows with compressible flow algorithms.

Van Leer, et.al. [1] recently developed an optimal, analytic preconditioning technique to reduce eigenvalue stiffness over the full Mach-number range. They proposed the method for first-order, perfect-gas flow simulations using explicit time integration and showed two-dimensional numerical results. Previously, preconditioning methods were proposed by among others Chorin [2], Turkel [3], Choi and Merkle [4], and Viviand [5]. While effective in their scope, preconditioning methods before that of Van Leer lacked both the physical connection with the fluid dynamics and the necessary robustness for all Mach-number flows.

Van Leer adopted an approach based upon wave propagation. The two-dimensional Euler equations exhibit a four wave structure consisting of entropy, vorticity, and acoustic waves. At supersonic speeds these waves travel in predictable Mach-number-dependent directions. With an understanding of these propagation directions, a preconditioning matrix can be determined that normalizes each wave's speed [1]. However, at subsonic speeds the acoustic waves travel omnidirectionally, and in [1] Van Leer uses the structure of the supersonic preconditioning matrix to obtain the optimal subsonic matrix. By multiplying the flux-balance residual with the preconditioning matrix, we can scale the acoustic wave speeds so that all waves propagate at the same rate, an essential property to eliminate inherent eigenvalue stiffness.

The numerical elements of flows with thermal and chemical non-equilibrium have been developed by among others Grossman and Cinnella [6], Grossman and Walters [7], Vinokur and Liu [8], Glaister [9], and Liou et.al. [10]. They developed fux-split algorithms for fluid-dynamic simulations with chemical production and vibrational-energy relaxation processes. Walters et.al. $[11,12]$ implemented these algorithms in developing a production-level computational code, the General Aerodynamic Simulation Program (GASP). In GASP, stiffness from competing chemical and fluid-dynamic time scales are effectively neutralized by treating the chemical source terms implicitly.

This paper synthesizes the thermo-chemical nonequilibrium flux-splitting of Grossman and Cinnella and the characteristic wave preconditioning of Van Leer into a powerful tool for implicitly solving two and threedimensional flows with generalized finite-rate chemistry. Proof of its effectiveness in a real-gas flow regime is given for a very-high-temperature diverging nozzle. However, the majority of our results are for the incompressible perfect-gas regime where the most dramatic acceleration is attained.

The following section describes the stream-aligned coordinate system from which the preconditioning matrix and flux function are derived. In section 3 we describe the preconditioning matrix for generalized finiterate chemistry, the implicit formulation, and the nu- 
merical implementation of these ideas. Next, we explain the necessary modification to the flux function for preconditioning. In section 5 we provide insight into the implicit damping qualities of the one-dimensional Euler equations with and without preconditioning. In addition the penalty of using a standard flux formula with preconditioning is analytically illustrated. Next, we provide numerical results. An inviscid channel flow is used to determine the convergence-rate performance of the preconditioning at all Mach numbers and for several Courant numbers. For the transonic case, the effect of higher-order accuracy through MUSCL differencing is determined. Further results include a verylow-speed Eppler 387 airfoil. Space marching cases include a supersonic three-dimensional wedge and an axisymmetric nozzle with chemical reactions. Finally, in section 7 we describe the pitfalls and resolutions to preconditioning the one-dimensional Navier-Stokes equations. Viscous test cases include the internal structure of a normal shock. Conclusions and future work for the full Navier-Stokes equations appear in the closing section.

\section{Stream-aligned Coordinate Systems}

Before discussing the finite-rate extension of matrix preconditioning, the framework for analyzing the two-dimensional Euler equations is set. The uninitiated reader is referred to the earlier works by Van Leer, et.al. [1], Lee [17] and Turkel [3,19] as an introduction. Feng and Merkle [26] give a thorough review of the preconditionings used before that of Van Leer.

The underlying physics of fluid dynamics leap forward from the symmetric linearized form of the Euler equations. To obtain this perceptive form, we conduct coordinate transformations to change from Cartesian conservation variables to stream-aligned symmetrization variables. The resulting system quickly reveals the primary wave systems dictating the Euler equations. A secondary motive for the transformation is to exchange the cumbersome inviscid flux Jacobians, $\partial f / \partial Q$ and $\partial g / \partial Q$, for simpler forms. The resulting Jacobians are then much easier to manipulate.

First let's start at the conservation level. The wellknown, two-dimensional Euler equations in conservation form are

$$
\frac{\partial Q}{\partial t}+\frac{\partial f}{\partial x}+\frac{\partial g}{\partial y}=0
$$

where

$$
Q \equiv\left\{\begin{array}{c}
\rho \\
\rho u \\
\rho v \\
\rho e_{0}
\end{array}\right\}, f \equiv\left\{\begin{array}{c}
\rho u \\
\rho u^{2}+p \\
\rho u v \\
\rho u h_{0}
\end{array}\right\}, g \equiv\left\{\begin{array}{c}
\rho v \\
\rho v u \\
\rho v^{2}+p \\
\rho v h_{0}
\end{array}\right\} .
$$

For a perfect gas, the equations are closed by the perfect-gas equation of state

$$
p=(\gamma-1) \rho e .
$$

In the equations above $\rho, u, v, e_{0}, p, h_{0}, \gamma$ are the density, $(x, y)$ components of velocity, stagnation internal energy, pressure, stagnation enthalpy and ratio of specific heats, respectively.

The variables, $U$, that symmetrize the inviscid flux Jacobians are known in differential form as

$$
d U \equiv\left\{\begin{array}{c}
d p /(\rho a) \\
d u \\
d v \\
d p-a^{2} d \rho
\end{array}\right\}
$$

Linearization of the Euler equations with respect to the symmetrization variables yields

$$
\frac{\partial U}{\partial t}+A^{\prime} \frac{\partial U}{\partial x}+B^{\prime} \frac{\partial U}{\partial y}=0
$$

where

$$
\begin{aligned}
& A^{\prime} \equiv \frac{\partial U}{\partial q} \frac{\partial q}{\partial Q} \frac{\partial f}{\partial Q} \frac{\partial Q}{\partial q} \frac{\partial q}{\partial U}, \\
& B^{\prime} \equiv \frac{\partial U}{\partial q} \frac{\partial q}{\partial Q} \frac{\partial g}{\partial Q} \frac{\partial Q}{\partial q} \frac{\partial q}{\partial U},
\end{aligned}
$$

Note that from Equ. (4) we can identify the Jacobian of $U$ with respect to the primitive variables, $q=$ $(\rho, u, v, p)^{T}$ which is

$$
\frac{\partial U}{\partial q}=\left[\begin{array}{cccc}
0 & 0 & 0 & 1 /(\rho a) \\
0 & 1 & 0 & 0 \\
0 & 0 & 1 & 0 \\
-a^{2} & 0 & 0 & 1
\end{array}\right]
$$

The Jacobian of the conservative variables with respect to the primitive variables assuming a perfect-gas law are

$$
\frac{\partial Q}{\partial q}=\left[\begin{array}{cccc}
1 & 0 & 0 & 0 \\
u & \rho & 0 & 0 \\
v & 0 & \rho & 0 \\
\left(u^{2}+v^{2}\right) / 2 & \rho u & \rho v & 1 /(\gamma-1)
\end{array}\right],
$$

The converse Jacobian is simply the inverse of the above matrix, or

$$
\frac{\partial q}{\partial Q}=\left(\frac{\partial Q}{\partial q}\right)^{-1}
$$

All of these transformation matrices are fairly trivial, and using them, the inviscid flux Jacobians transform into the matrices $A^{\prime}$ and $B^{\prime}$ of Equ. (6) as

$$
A^{\prime}=\left[\begin{array}{llll}
u & a & 0 & 0 \\
a & u & 0 & 0 \\
0 & 0 & u & 0 \\
0 & 0 & 0 & u
\end{array}\right]
$$


and

$$
B^{\prime}=\left[\begin{array}{llll}
v & 0 & a & 0 \\
0 & v & 0 & 0 \\
a & 0 & v & 0 \\
0 & 0 & 0 & v
\end{array}\right]
$$

The state vector's velocities are with respect to a Cartesian coordinate system, so we need to rotate the axes to the stream-aligned coordinate system. A transformation from the $(x, y)$ system to the streamaligned $(\xi, \eta)$ system through an angle, $\theta$, yields the transformation matrix

$$
\frac{\partial \vec{U}}{\partial U}=\left[\begin{array}{cccc}
1 & 0 & 0 & 0 \\
0 & \cos \theta & \sin \theta & 0 \\
0 & -\sin \theta & \cos \theta & 0 \\
0 & 0 & 0 & 1
\end{array}\right] .
$$

The angle $\theta$ is determined from the velocity components as $\tan \theta=v / u$. We also need to differentiate with respect to the stream-aligned coordinates. Applying the chain rule to $\partial U / \partial x$ and $\partial U / \partial y$, the final metamorphosis of the Euler equations is complete. The equations become

$$
\frac{\partial \bar{U}}{\partial t}+\bar{A} \frac{\partial \bar{U}}{\partial \xi}+\bar{B} \frac{\partial \bar{U}}{\partial \eta}=0
$$

where

$$
\bar{A}=\left[\begin{array}{llll}
q & a & 0 & 0 \\
a & q & 0 & 0 \\
0 & 0 & q & 0 \\
0 & 0 & 0 & q
\end{array}\right]
$$

and

$$
\bar{B}=\left[\begin{array}{llll}
0 & 0 & a & 0 \\
0 & 0 & 0 & 0 \\
a & 0 & 0 & 0 \\
0 & 0 & 0 & 0
\end{array}\right]
$$

The eigenvalues and underlying wave structure are evident in this simple form, and the flux Jacobians are now trivial. The derivations of the preconditioning matrix and modified flux function start with this streamaligned system. For numerical implementation, the preconditioning matrix must be transformed back to update the desired state variables which are usually the conserved variables with Cartesian velocities. Later, in this paper we document the preconditioning matrix for updating primitive variables. The artificial-viscosity matrix in the modified flux function must be transformed to a cell-face-aligned system with conservation variables for finite-volume implementation.

\section{Preconditioning and Implicit Formulation}

The proper preconditioning matrix in two dimensions results from a wave analysis of the governing hyperbolic equations. Upon linearization, the eigenvalues

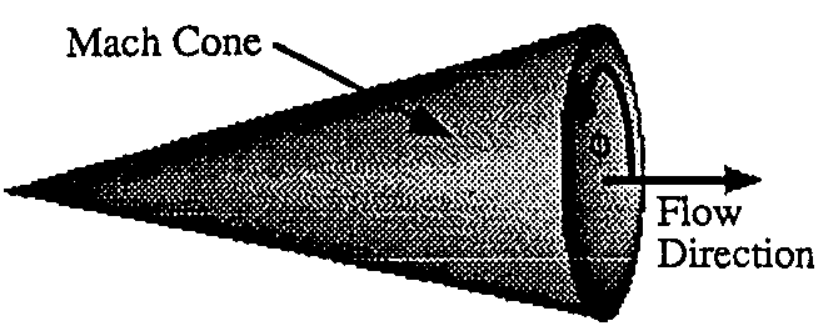

Figure 1. Supersonic three-dimensional Mach cone illustrating angular variable, $\phi$.

of the inviscid flux Jacobians determine the wave speeds of the system through

$$
\bar{A} \cos \theta+\bar{B} \sin \theta \Rightarrow \lambda(\theta) \text {. }
$$

The true character of the equations comes from the wave fronts. With the wave speeds, $\lambda(\theta)$, and their derivatives, $\lambda^{\prime}(\theta)$, we can determine the wave fronts by

$$
\left\{\begin{array}{l}
x \\
y
\end{array}\right\}=\left[\begin{array}{rr}
\cos \theta & -\sin \theta \\
\sin \theta & \cos \theta
\end{array}\right]\left\{\begin{array}{l}
\lambda(\theta) \\
\lambda^{\prime}(\theta)
\end{array}\right\}
$$

Normalizing the envelope of wave fronts gives the preconditioning matrix which multiplies a flux balance residual. For Equ. (1), the preconditioner serves as a filter and multiplies the residual as follows

$$
\frac{\partial Q}{\partial t}=-(\mathcal{P R E})\left(\frac{\partial f}{\partial x}+\frac{\partial g}{\partial y}\right)
$$

In the stream-aligned system with symmetrization variables as determined in Section 2, the twodimensional preconditioning matrix, $P$, that optimally normalizes the acoustic and convective wave fronts is

$$
P=\left[\begin{array}{cccc}
\frac{\tau}{\beta^{2}} M^{2} & -\frac{\tau}{\beta^{2}} M & 0 & 0 \\
-\frac{\tau}{\beta^{2}} M & \frac{\tau}{\beta^{2}}+1 & 0 & 0 \\
0 & 0 & \tau & 0 \\
0 & 0 & 0 & 1
\end{array}\right]
$$

The parameters, $\tau$ and $\beta$, are determined with the Mach number by

$$
\begin{aligned}
& \beta= \begin{cases}\sqrt{M^{2}-1}, & \text { if } M \geq 1 ; \\
\sqrt{1-M^{2}}, & \text { if } M<1,\end{cases} \\
& \tau= \begin{cases}\sqrt{1-M^{-2}}, & \text { if } M \geq 1 ; \\
\sqrt{1-M^{2}}, & \text { if } M<1 .\end{cases}
\end{aligned}
$$

Lee's doctoral thesis [17] is an excellent and highly recommended reference for the details of this matrix derivation.

The three-dimensional preconditioning matrix must equalize all the acoustic waves in an identical way. This 


\begin{tabular}{|c|c|c|c|}
\hline $\begin{array}{c}\text { Mach No. } \\
\text { Range }\end{array}$ & $\begin{array}{l}\text { Cond. No. } \\
\text { (Euler) }\end{array}$ & $\begin{array}{l}\text { Cond. No. } \\
\text { (Pre. Euler) }\end{array}$ & $\begin{array}{l}\text { Cond. No. } \\
\text { Ratio }\end{array}$ \\
\hline $\begin{array}{c}0<M \leq 1 / 2 \\
1 / 2 \leq M<1 \\
(2-D \text { or } 3-D)\end{array}$ & $\begin{array}{l}\frac{1+M}{M} \\
\frac{1+M}{1 \rightarrow M}\end{array}$ & $\frac{1}{\sqrt{1-M^{2}}}$ & $\begin{array}{l}\frac{(1+M) \sqrt{1-M^{2}}}{M} \\
\frac{(1+M) \sqrt{1-M^{2}}}{1-M}\end{array}$ \\
\hline $\begin{array}{l}M>1(2-\mathrm{D}) \\
M>1(3-\mathrm{D})\end{array}$ & $\frac{M \pm 1}{M-1}$ & $\begin{array}{c}1 \\
\frac{M}{\sqrt{M^{2}-1}}\end{array}$ & $\begin{array}{c}\frac{M+1}{M-1} \\
\frac{M+1}{M} \sqrt{\frac{M+1}{M-1}}\end{array}$ \\
\hline
\end{tabular}

Table 1. Theoretical convergence-rate acceleration based on the partial differential equations' condition numbers.

requires that the wave speeds, which are now propagating in three dimensions instead of the restricted two dimensional plane, have no angular dependence, $\phi$, in the cross-flow plane, Fig. 1. The only preconditioning matrix with the form of Equ. (19) that satisfies this independence criterion is

$$
P=\left[\begin{array}{ccccc}
\frac{\tau}{\beta^{2}} M^{2} & -\frac{\tau}{\beta^{2}} M & 0 & 0 & 0 \\
-\frac{\tau}{\beta^{2}} M & \frac{\tau}{\beta^{2}}+1 & 0 & 0 & 0 \\
0 & 0 & \tau & 0 & 0 \\
0 & 0 & 0 & \tau & 0 \\
0 & 0 & 0 & 0 & 1
\end{array}\right]
$$

Unfortunately, now $\tau$ multiplies and shortens a streamwise shear wave speed penalizing the supersonic condition number.

The theoretical acceleration in convergence rate is the ratio of the condition numbers with and without preconditioning. A summary of the subsonic and supersonic condition numbers and corresponding theoretical acceleration rates is given in Table 1 . The minimum theoretical speed-up for subsonic flow is $3 \sqrt{3} / 2$ and occurs at a Mach number of $M=0.5$. For supersonic flow, the benefit of preconditioning decreases as the Euler waves grow closer together with increasing Mach number.

For finite-rate chemistry, the state vector of unknowns is variable in length. Therefore, the preconditioning matrix extended to generalized finite-rate chemistry must accommodate a flexible system of moving waves. Fortunately, no new kind of wave appears in the system. We have only entropy and vorticity waves moving with the fluid and acoustic waves propagating in Mach-number dependent directions. The nonequilibrium vibrational energies and mass fractions act strictly as convective waves. The variables, $U$, which symmetrize the inviscid flux Jacobians are defined for generalized finite-rate chemistry in differential form as

$$
d U=\left\{\begin{array}{c}
d p /(\rho a) \\
d p-\left(\rho a^{2} / \rho_{2}\right) d \rho_{2} \\
\vdots \\
d p-\left(\rho a^{2} / \rho_{N}\right) d \rho_{N} \\
d u \\
d v \\
d w \\
d p-\left(\rho a^{2} / \rho_{1}\right) d \rho_{1}-\left(\rho a^{2} / e_{n_{1}}\right) d e_{n_{1}} \\
\vdots \\
d p-\left(\rho a^{2} / \rho_{M}\right) d \rho_{M}-\left(\rho a^{2} / e_{n_{M}}\right) d e_{n_{M}} \\
d p-\left(\rho a^{2} / \rho_{1}\right) d \rho_{1}
\end{array}\right\}
$$

Note that this matrix reduces to the symmetrization variables of Equ. (4) for two-dimensional flow of a perfect gas. The same derivation that generates the preconditioning matrix in Equ. (21) yields the generalized finite-rate preconditioner. This matrix is given in Appendix A. The governing equations for generalized finite-rate chemistry are given in Walters, et.al. [11].

\section{Explicit Impiementation}

As stated previously, the preconditioning matrix is derived in a coordinate system that is not appropriate for numerical implementation. To use the above preconditioning matrix, we must transform back to a conservative system and update the state variables of our choice. Note that the state variables may be the conservative or primitive variables or any other system which contains the velocity components and two thermodynamic variables. This includes both the pressure and density based formulations. It's simply a matter of choice.

We choose to transform from stream-aligned, symmetrization variables to Cartesian, conservation varjables. This requires a rotation of the velocity axes and a variable transformation. Preconditioning the governing equations and using standard Euler explicit time integration results in the following numerical procedure.

$$
\Delta Q=-\frac{\Delta t}{V o l} \frac{\partial Q}{\partial q} \frac{\partial q}{\partial U} \frac{\partial U}{\partial \bar{U}} P \frac{\partial \bar{U}}{\partial U} \frac{\partial U}{\partial q} \frac{\partial q}{\partial Q} R\left(Q^{n}\right)
$$

To determine the time step, $\Delta t$, the CFL-like relaxation factor, $\nu$, is defined as

$$
\nu \equiv \frac{q \Delta t}{\Delta L} .
$$


The characteristic length, $\Delta L$, is any length that reduces to the one-dimensional definition for grid-aligned flow. Notice the absence of the sound speed in the above CFL definition. For the preconditioned equations the spectral radius is simply the convective velocity, and does not include the sound speed. This new definition of the CFL number is important for the proper time-step calculation in slow-flow regions. Simplifying the notation we define

$$
\mathcal{P} \equiv \frac{\partial q}{\partial U} \frac{\partial U}{\partial \bar{U}} P \frac{\partial \bar{U}}{\partial U} \frac{\partial U}{\partial q} .
$$

Appendix $B$ documents $\mathcal{P}$ for generalized finite-rate chemistry in three dimensions. If we update primitive variables, instead of conservative as in Equ. (23), the Euler explicit algorithm becomes

$$
\Delta q=-\frac{\Delta t}{V o l} \mathcal{P} \frac{\partial q}{\partial Q} R\left(q^{n}\right) .
$$

Implicit Implementation

For implicit time integration, we move the preconditioning matrix of Equ. (18) to the left hand side. Then, the system can be solved using any direct or indirect solver in the literature. Note that with very high time steps the effect of the preconditioning is canceled out. However, we can achieve significant improvements for low-speed flows and for simulations using approximate factorization. Standard Euler implicit time integration for the preconditioned equations yields the following formulation to update primitive variables:

$$
\left[\frac{V o l}{\Delta t} \frac{\partial Q}{\partial q} \mathcal{P}^{-1}+\frac{\partial R}{\partial q}\right]^{n} \Delta q=-R\left(q^{n}\right) \text {. }
$$

Appendix $\mathrm{C}$ documents $\mathcal{P}^{-1}$ for generalized finite-rate chemistry in three dimensions. The matrices $\partial Q / \partial q$ and $\partial q / \partial Q$ are given in Grossman and Cinnella [6].

\section{Modified Flux Formulas}

In developing flux formulas for the preconditioned Euler equations, the form of the numerical scheme must consistently represent the modeled partial differential equations. Therefore, the artificial viscosity for an approximate Riemann solver must be modified for preconditioning. For the original Roe algorithm, the artificialviscosity matrix is

$$
\text { A.V. }=|\hat{A}|=|A \cos \theta+B \sin \theta|,
$$

which consistently represents the linearized Euler equations. Here, $\cos \theta$ and $\sin \theta$ are the cell-face-normal direction cosines. The equivalent artificial-viscosity matrix for preconditioning must be

$$
|P \hat{A}|=|P(A \cos \theta+B \sin \theta)|
$$

and not

$$
P|\hat{A}|=P|A \cos \theta+B \sin \theta|
$$

to consistently depict the preconditioned Euler equations. For a robust artificial-viscosity matrix, a slight modification is still necessary. The matrix $|P \hat{A}|$ contains singular terms for certain flow angles, particularly the free-stream direction. An alternative stems from the multi-dimensional Riemann solver research and conservatively sums the fluxes from multiple Riemann problems. The alternative artificial-viscosity matrix is then

$$
\left.\left.|P \hat{A}|=\left|P A \cos \theta_{\bar{x}}\right|+\mid P B \cos \theta_{\bar{y}}\right)|+| P C \cos \theta_{\bar{z}}\right) \mid
$$

where $\theta_{\bar{z}}, \theta_{\bar{y}}$, and $\theta_{\bar{z}}$ are the direction cosines between the flow direction orthonormals and the cell face normal. The full equations are documented in Appendix $D$. The inverse of $P$ must premultiply the artificialviscosity matrix so that the modified Roe flux is preserved upon multiplication of the residual by the preconditioning matrix. The resulting modified flux becomes

$$
\hat{f}_{j+1 / 2}=\frac{1}{2}\left(\hat{f}_{j}+\hat{f}_{j+1}\right)-\frac{1}{2} P^{-1}|P \hat{A}|\left(Q_{j+1}-Q_{j}\right)
$$

Appendix D contains the modified Roe flux for generalized finite-rate chemistry.

\section{Numerical Analysis}

\section{Euler Implicit Damping Characteristics}

In this section, we retreat briefly to the onedimensional Euler equations. From within this simple framework, the deficiencies of a typical compressible code can be seen. We analyze Euler implicit time integration with the preconditioning algorithms proposed above. Of course, we include the standard nonpreconditioned scheme as a comparison.

Recall that a discrete grid restricts the representative range of wavelengths and frequencies. The smallest wavelength and corresponding highest frequency that can be depicted on a finite discretization are $\lambda=2 \Delta x$ and $\beta_{x}=\pi$; respectively. The wavelength, wave number, and frequency are related through the following:

$$
\lambda=\frac{2 \pi}{m}=\frac{2 \pi}{\beta_{x}} \Delta x .
$$

We are interested in the spectral radius at both long and short wavelengths, (i.e. $\beta_{x} \rightarrow 0$ and $\beta_{x} \rightarrow \pi$ ) because typically, during convergence to the steady state, short waves decay quickly while long waves persist, expiring gradually. 


\section{Local Time Stepping}

We want to determine the damping behavior of the implicit time operator over the entire Mach number range. The damping is determined by the gain matrix from a Von Neumann stability analysis. We want to determine the spectral radius, and thus the amplification factor, of the update matrix. This matrix is a function of the Mach number and the Fourier frequency.

Performing a Von Neumann stability analysis of the Euler implicit procedure with Roe flux-difference splitting produces an amplification matrix whose inverse is given below.

$$
G^{-1}=I+\frac{2 \lambda}{M+1}|A| \sin ^{2} \frac{\beta_{x}}{2}+i \frac{\lambda}{M+1} A \sin \beta_{x} .
$$

The Courant number is defined as $\lambda \equiv(u+a) \Delta t / \Delta x$. The smallest eigenvalue will be the inverse of the spectral radius. The three analytic eigenvalues of the inverse gain matrix are

$$
\begin{aligned}
& \lambda_{-}=1+\frac{2 \lambda|M-1|}{M+1} \sin ^{2} \frac{\beta_{x}}{2}+i \frac{\lambda|M-1|}{M+1} \sin \beta_{x}, \\
& \lambda_{u}=1+\frac{2 \lambda M}{M+1} \sin ^{2} \frac{\beta_{x}}{2}+i \frac{\lambda M}{M+1} \sin \beta_{x}, \\
& \lambda_{+}=1+2 \lambda \sin ^{2} \frac{\beta_{x}}{2}+i \lambda \sin \beta_{x} .
\end{aligned}
$$

A carpet plot of the spectral radii over a Mach number range of $10^{-2}$ to 10 and Fourier frequencies from lowest to highest is given in Fig. 2. Notice that the spectral radius approaches unity in the limit of $M \rightarrow 0$ for all wavelengths and equals unity at exactly Mach 1. A nearly unitary gain results in minimal damping of a solution error in these flow regimes. Notice that significant damping occurs for supersonic and moderate subsonic Mach numbers for the high frequency waves. However, as the grid points in a simulation increase in number, longer wavelengths are numerically represented (i.e. $\beta_{x} \rightarrow 0$ ) and their gain approaches unity.

\section{Characteristic Time Stepping}

Von Neumann stability analysis with preconditioning and the modified Roe flux produces an amplification matrix whose inverse is

$$
G^{-1}=I+\frac{2 \nu}{M}|P A| \sin ^{2} \frac{\beta_{x}}{2}+i \frac{\nu}{M} P A \sin \beta_{x}
$$

where $\nu \equiv u \Delta t / \Delta x$. The three eigenvalues of the inverse are identical and are

$$
\lambda_{-}=\lambda_{u}=\lambda_{+}=1+2 \nu \sin ^{2} \frac{\beta_{x}}{2}+i \nu \sin \beta_{x}
$$

Notice the absence of the Mach number in the above equation. More specifically, we have the same

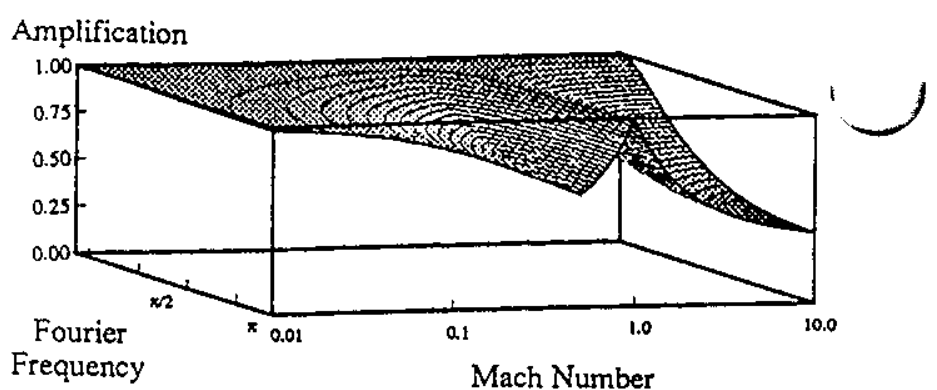

Figure 2. Gain over a range of Mach numbers for the Euler equations with first-order Roe flux-difference splitting using Euler implicit time integration and $\lambda=$ 1.0 .

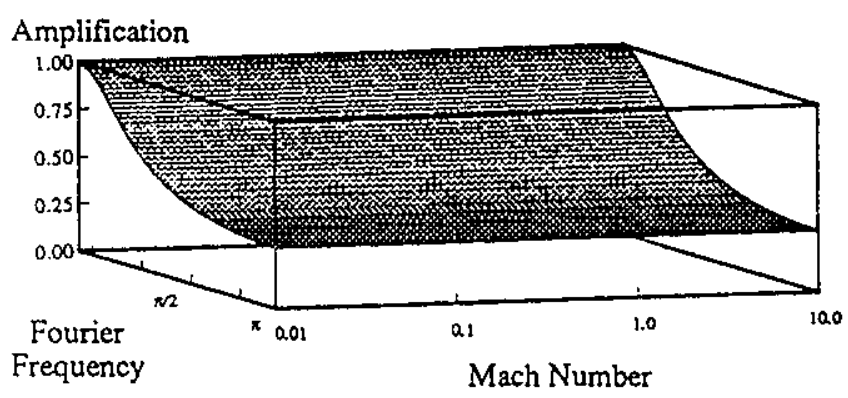

Figure 3. Gain over a range of Mach numbers for the preconditioned Euler equations with first-order modified Roe flux-difference splitting using Euler implici ${ }^{+}$ time integration and $\nu=1.0$.

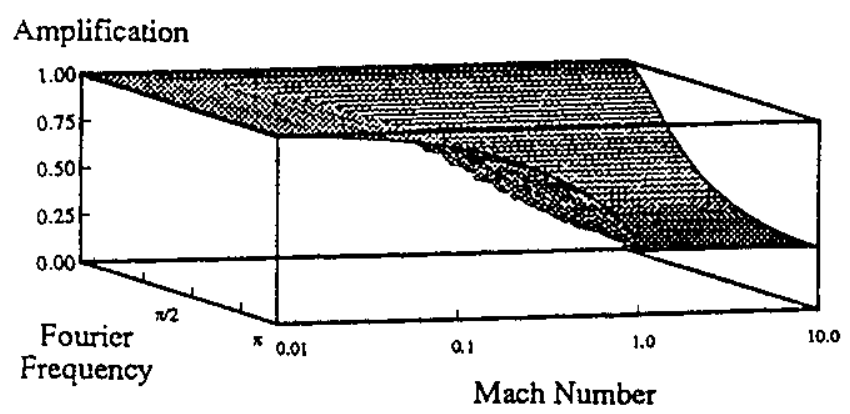

Figure 4. Gain over a range of Mach numbers for the preconditioned Euler equations with first-order standard Roe flux-difference splitting using Euler implicit time integration and $\nu=1.0$.

damping characteristics at all Mach numbers as the scalar wave equation. This is precisely our goal. A carpet plot of the gain for the range of frequencies and the same representative range of Mach numbers is illustrated in Fig. 3. We see significant improvement from the damping shown in Fig. 2, which is the current state of the art.

One should be careful. If the standard Roe algo rithm is preconditioned without any flux modificatio. 
as in Equ. (30), the spectral radius degrades in the subsonic region for all frequencies as shown in Fig. 4. When treated implicitly, this formulation is unconditionally stable, yet the gain approaches unity as $M \rightarrow 0$, and, thus, we have poor subsonic damping. Using an explicit time marching strategy, the same algorithm requires a prohibitively small time step for stability [1]. The modified Roe flux is necessary for preconditioning subsonic flows.

\section{Numerical Results}

Channel Flow

\section{Objectives}

First, we demonstrate the abilities of the preconditioning to accelerate convergence using a simple geometry. The transonic flow through a channel with a circular-arc disturbance on the lower wall was the subject of a GAMM workshop in 1979 [20]. The transonic flow is slow to converge because the transonic shock which forms on the recompression side of the arc is reluctant to find its final steady state position.

Using this problem, we can determine the answers to many questions: How well does the preconditioning accelerate the convergence? What happens to convergence for different flux functions? What is the optimal Courant number to use with approximate factorization and, in particular, is this optimal value the same with and without preconditioning? What is the effective spectral radius of the iteration scheme? How does higher-order differencing change the performance of the preconditioned equations? How does the preconditioning perform across the entire subsonic Mach number range? And, finally, how accurate are the computed incompressible solutions, which we can compare to the small-disturbance potential equation?

\section{Numerical Synopsis}

The circular arc is nestled in the lower wall and has unit chord. The inflow and outflow boundaries are two chords away from the leading and trailing edges, and the top wall is 2.073 chords above the lower wall. The curvature-corrected symmetry technique of Dadone and Grossman [23] is used as our tangency boundary condition on the upper and lower walls. At the inflow and outflow boundaries, the corresponding left and right states are set at the free-stream value. A numerical flux is calculated at all boundaries which alleviates boundary condition stiffness from the newly modified Roe flux function. We integrate in time using approximate factorization, and use both Van Leer flux-vector splitting and Roe flux-difference splitting.

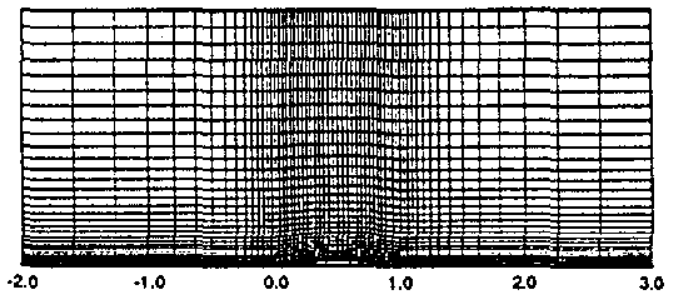

Figure 5. Typical mesh used to simulate the flow through a channel. All pressure contour plots are presented on this $61 \times 31$ grid.

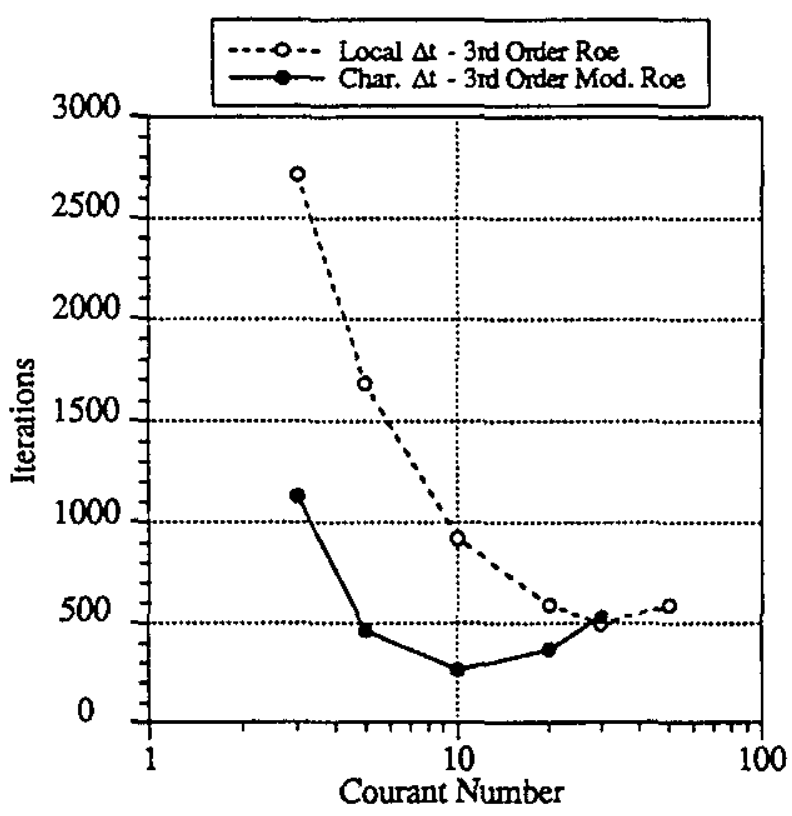

Figure 6. Courant-number study using approximate factorization and Roe flux-difference splitting for the transonic channel.

Four different grids of dimension $31 \times 16,61 \times 31$, $91 \times 61$, and $181 \times 91$ are used. All pressure contour plots are given on the $61 \times 31$ mesh shown in Fig. 5 . The Courant-number study below was done on the coarsest mesh to conserve excessive CPU time.

\section{Courant-Number Study}

The transonic flow solution has a free-stream Mach number of $M=0.85$. Convergence of a flux balance residual to machine zero on a Cray YMP is obtained using MUSCL differencing $(\kappa=1 / 3)$ and the Van Albada limiter. The results for the range of time steps is shown in Fig. 6.

First, notice the typical behavior of the approximate factorization algorithm (i.e. gains that approach unity for both small and large Courant numbers and an optimal Courant number in the middle on the order of 10). The Courant number corresponding to the 


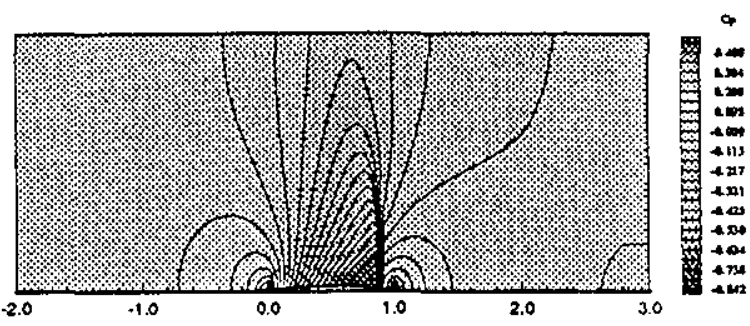

Figure 7. Pressure coefficient contours for the transonic $M=0.85$ solution.

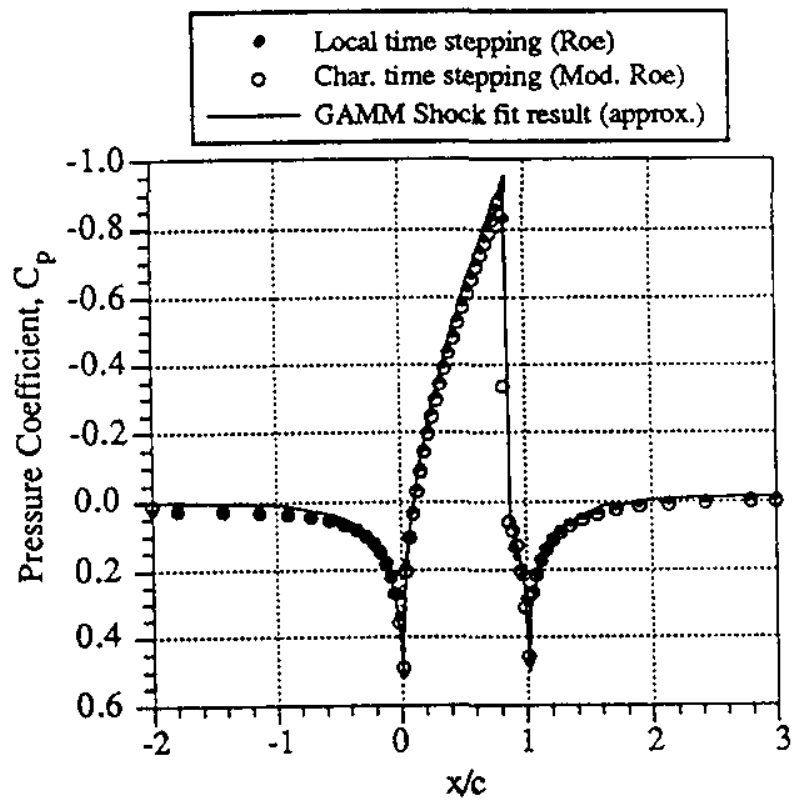

Figure 8. Lower wall pressure coefficient for the transonic flow through a channel compared to the shock-fit results of [20].

minimum number of iterations appears to be different for local time stepping than for characteristic time stepping; however, the definition of Courant number differs between the two. The lowest number of iterations required to solve the transonic flow using fluxdifference splitting is 496 with local time stepping and 263 with characteristic time stepping. The optimal Courant number for local time stepping is not necessarily the proper selection for characteristic time stepping. The first-order results are similar.

Pressure contours are shown in Fig. 7, and the lower wall distribution is shown in Fig. 8 . The result from Rizzi's shock-fitting code [20] is included as a nearly exact solution. Careful examination will show that the peak pressure using the modified Roe algorithm is lower than for standard flux-difference splitting. This can be attributed to a larger artificial viscosity associated with the modified fux algorithm.

\section{Numerical or Effective Spectral Radius}

In this sub-section, the residual histories of a gridconvergence cycle are used to determine the spectral radius of the numerical algorithm. After initial short wavelengths are smoothed, the residual history reflects the gain of the longest numerically achievable wavelength. The dominant long wave makes a residual history on a semi-log plot appear linear. The spectral radius for this one wave can be estimated strictly by examining the residual history of this final damping region. If the error at iteration number $n$ is a fraction (i.e. the spectral radius) of the error at iteration number $n+1$, or if

$$
\epsilon^{n+1}=\rho_{s} \epsilon^{n},
$$

then the spectral radius over several iterations numbered $n$ to $N$ is

$$
\rho_{s}=\left[\frac{\left\|R^{N}\right\|_{2}}{\left\|R^{n}\right\|_{2}}\right]^{\frac{1}{N-n}} .
$$

A typical residual history for the transonic channel flow is shown in Fig. 9., and the corresponding spectral radii over several mesh refinements are shown in Fig. 10. As the grid spacing becomes finer, the long waves are linearly less damped, and consequently iterations and computational time increase. Note that the spectral radius for the characteristic time-stepping algorithm is lower for all mesh sizes in comparison to local time stepping. This indicates that a solution using preconditioning may be obtained on a grid with roughly twice as many points in each direction in the same number of iterations (i.e. same spectral radius).

\section{Mach-Number Study}

In this sub-section we investigate the performance of the preconditioning algorithm over a range of subsonic Mach numbers. Performance for preconditioning and local time stepping using Roe's scheme is shown for first and higher order in Fig. 11.

The preconditioning algorithm gives convergence in fewer iterations for all free-stream Mach numbers. We have successfully run at Mach numbers as low as $M=10^{-8}$ while the solution using the standard Roe algorithm with local time stepping was entirely inaccurate, as will be demonstrated in the next sub-section.

Performance for the flux-vector splitting scheme is shown for first and higher order in Fig. 12. The mass splitting technique poorly models the Riemann problem for low Mach numbers and thus mathematically confuses the wave-based preconditioning matrix. Consequently, preconditioning with flux-vector splitting at low Mach numbers is unwise. The cross-over Mach number appears to be around $M \approx 0.5$. 


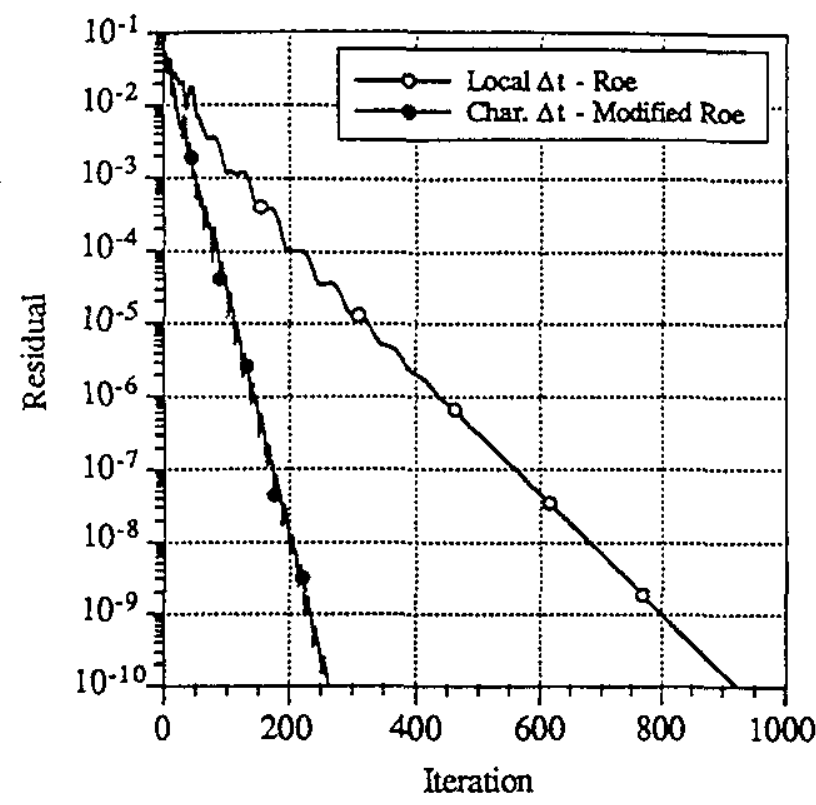

Figure 9. Residual history using approximate factorization with Roe flux-difference splitting for a transonic channel.

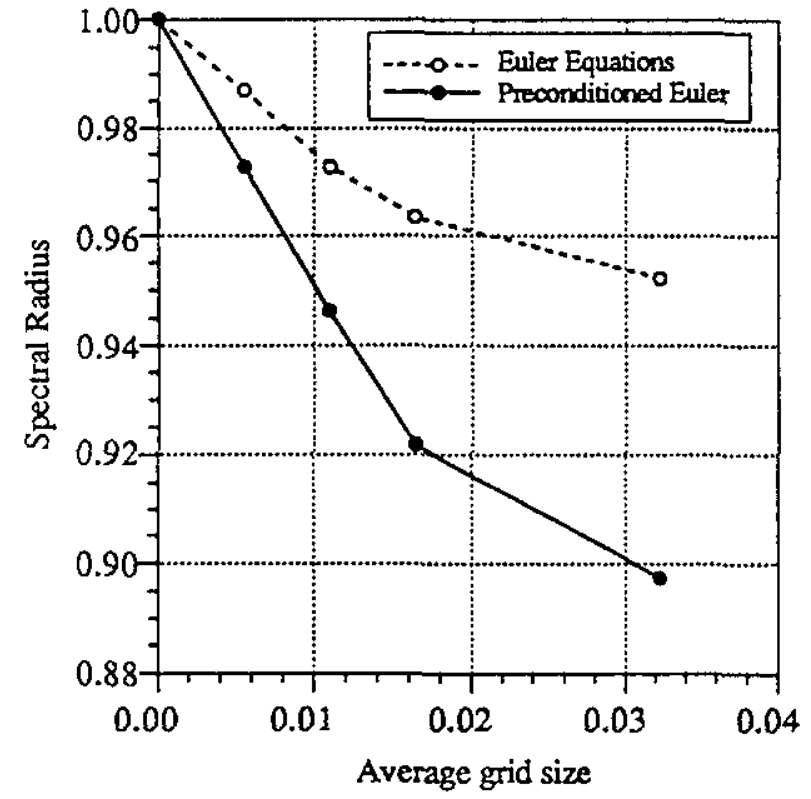

Figure 10. Spectral radius using approximate factorization with Roe flux-difference splitting for a transonic channel. The average grid size is one over the streamwise grid dimension.

To determine the result of preconditioning with higher-order interpolation, we calculate the percent savings over the Mach-number range for different accuracies and compare with the theoretical savings. Results are shown in Fig. 13. The savings were calculated as the difference between the required number

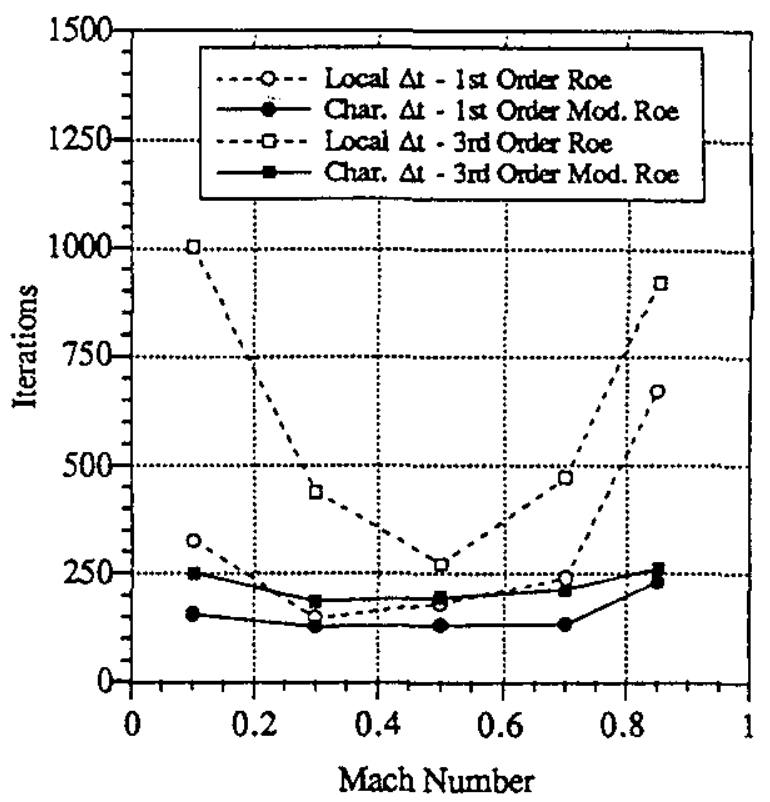

Figure 11. Convergence profile over subsonic Machnumber range using first and third-order MUSCL differencing with Roe flux-difference splitting.

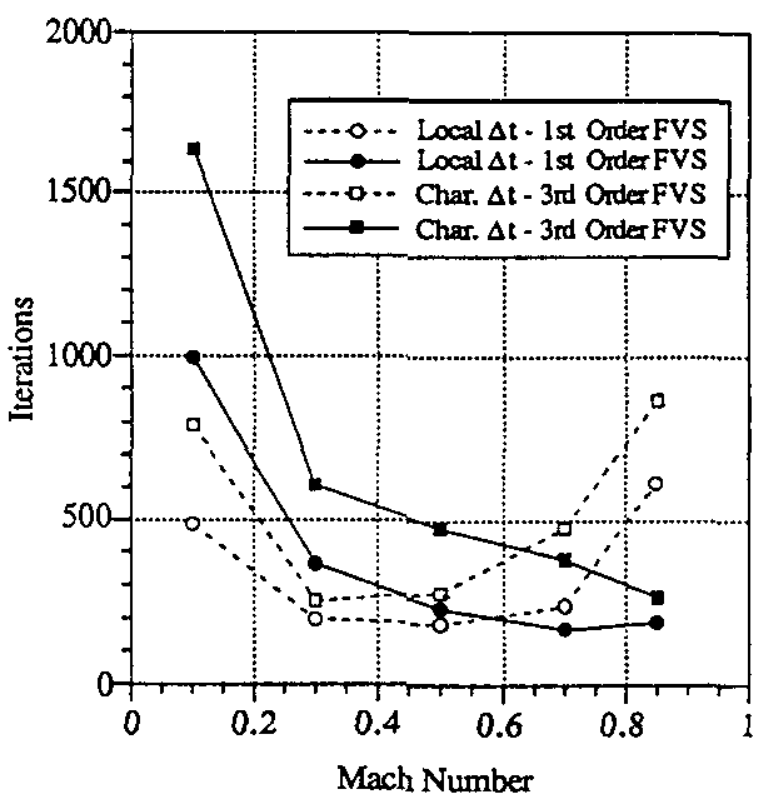

Figure 12. Convergence profile over subsonic Machnumber range using first and third-order MUSCL differencing with Van Leer flux-vector splitting.

of iterations divided by the iterations required without preconditioning. Preconditioning with higher-order interpolation is more efficient simply because the partial. differential equations are modeled more accurately.

Very-Low-Speed $\left(M=10^{-3}\right)$ Flow

An essential consideration for low Mach-number 


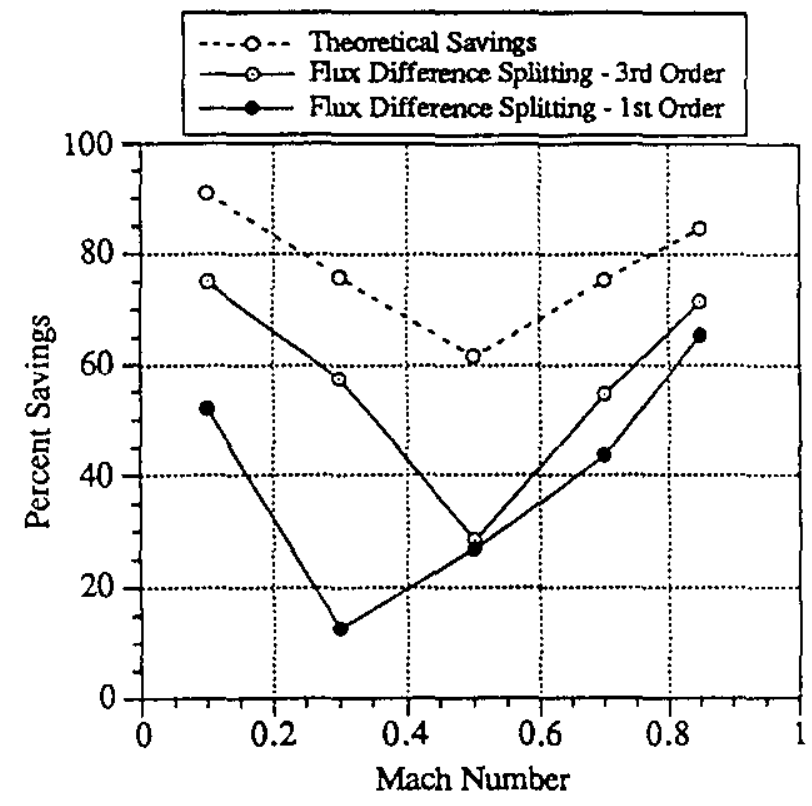

Figure 13. Percent savings using preconditioning over subsonic Mach-number range using Roe flux-difference splitting.

Hows is accurate pressure interpolation. The ratio of the convective to pressure terms is $1 /\left(\gamma M^{2}\right)$. This ratio approaches infinity at low Mach numbers. To accurately balance the flux of momentum, the pressure must be scaled to be on the order of the convective terms. Past researchers have incorporated a gauge pressure referenced to the free-stream pressure. Interpolation and flux evaluation is done with this small pressure perturbation. Summing the gauge pressure and free-stream pressure recovers the absolute pressure through

$$
p=p_{\infty}+p^{\prime}
$$

We have successfully utilized this strategy modeling very-low-speed flows. Note that to be perfectly consistent, we must scale the internal energy as suggested by Feng and Merkle [26]. For a perfect gas this is simply

$$
\rho e=(\rho e)_{\infty}+(\rho e)^{\prime}
$$

where

$$
(\rho e)_{\infty}=\frac{p_{\infty}}{\gamma-1} .
$$

However, for a flow code which models both perfect-gas and finite-rate chemistry, a gauge energy is impractical and was not done in the present computations. The consequence of this is seen in the residual history shown in Fig. 14. For very small residuals the energy balance exceeds the machine's precision. Increasing the machine precision or using a gauge energy allows convergence to higher orders.

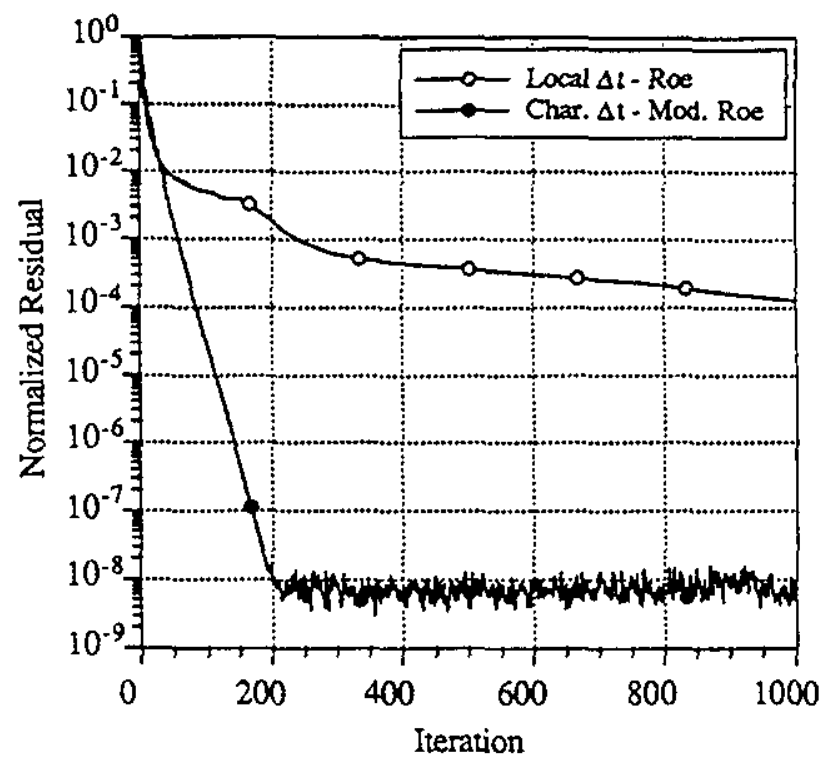

Figure 14. Very-low-speed channel flow residual history using approximate factorization and Roe fluxdifference splitting.

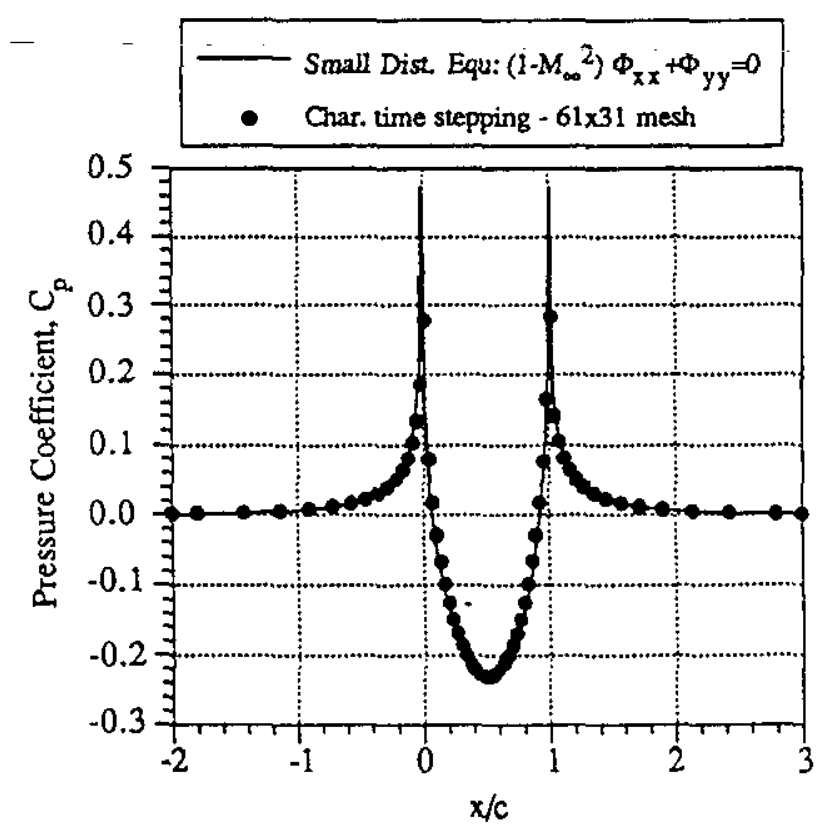

Figure 15. Pressure coefficient for the very-low-speed flow over a circular-arc airfoil.

The same channel geometry was run with a freestream Mach number of $M=0.001$. The pressure coefficient on the lower wall is compared to the smalldisturbance solution in Fig. 15. Thirty levels of pressure coefficient contours are shown in Fig. 16. Notice the symmetry in the data and in the contours stemming from the curvature-corrected symmetry boundary technique. An alternative solution using precondition- 


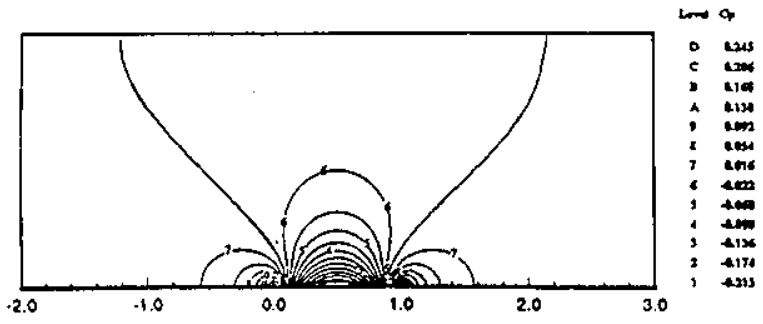

Figure 16. Pressure coefficient contours for very-lowspeed flow through a channel using preconditioning and a modified Roe flux.

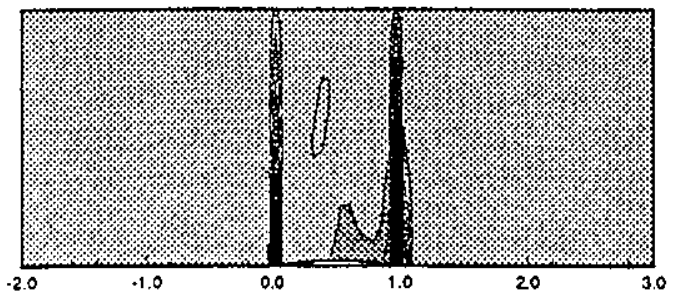

Figure 17. Pressure coefficient contours for very-lowspeed flow through a channel using preconditioning and a standard Roe flux.

ing with a standard Roe scheme is shown in Fig. 17. Obviously the modified Roe flux is essential for reasons of both accuracy and stability.

\section{Eppler 387 Airfoll}

An Eppler 387 airfoil was simulated at a freestream Mach number of $M=0.05$ and angle of attack, $\alpha=2^{\circ}$. There is little hope of accurately and efficiently calculating this flow with a standard compressible Euler code without including the preconditioning technique. A predominant flow phenomenon of this airfoil is the laminar separation bubble over most of the upper surface at low Reynolds number. This case typifies where we are with preconditioning and where we want to go. At present, we cannot simulate the complete NavierStokes equations and the laminar separation bubble using preconditioning. The first step toward this goal is given at the end of this paper for one-dimensional Navier-Stokes equations.

The computation used the $61 \times 41$ mesh shown in Fig. 18. To verify our inviscid results, we compared to a panel method. The pressure coefficient contours of the flow field are shown in Fig. 19, and the surface pressure is compared to wind tunnel data in Fig. 20. We obtained convergence of the normalized flux residual to a tolerance of six orders in 708 iterations and 81 CPU seconds. We iterated using the mesh sequencing capabilities of GASP and three levels of grid refinement.

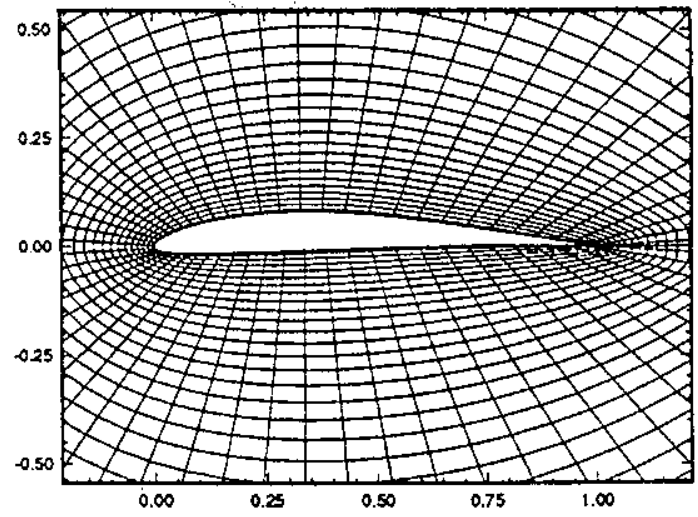

Figure 18. Close-up of the $61 \times 410$-mesh surrounding the Eppler 387 airfoil.

\section{Space Marching}

As a three-dimensional test case for the flux function and preconditioning matrix we simulate a skewed wedge that was proposed by Rumsey, et.al. [21] for their five-wave multi-dimensional Riemann solver. The inflow Mach number is 2.8 , and the geometry with pressure contours is shown in Fig. 21. The number of iterations required to converge an absolute residual by six orders per cross-flow plane is shown in Fig. 22. Approximate factorization is used in the cross flow plane with a non-linear residual update in the marching direction and a Courant number of five. Based on the condition numbers for three-dimensional $M=2.8$ flow, we would expect 1.97 times better convergence rate. In practice, the preconditioned case converges 0.93 to 1.33 times faster than without preconditioning depending upon the cross-flow plane.

The second marching case is the axi-symmetric reacting flow through a diverging nozzle. This case helps to determine the effect of preconditioning for flow with equilibrium and finite-rate chemistry as well as nonequilibrium vibrational relaxation. Four cases are run with different assumptions upon the thermodynamics. All cases use a Courant number of ten.

The inflow temperature is a balmy $9000 \mathrm{~K}$ and the inflow velocity is $3000 \mathrm{~m} / \mathrm{s}$ yielding an approximately sonic inflow Mach number. We expect large benefits from preconditioning within this sonic flow regime. Perfect gas, equilibrium, and finite-rate chemistry with and without non-equilibrium vibration were run using the Kang and Dunn [28] air chemistry model. The Mach number, temperature and mass fraction of diatomic nitrogen are shown in Figs. 23, 24 and 25. Iterations per plane are shown for the above cases in Figs. 26, 27 and 28. As the supersonic flow accelerates the condition number disparity becomes less, and 


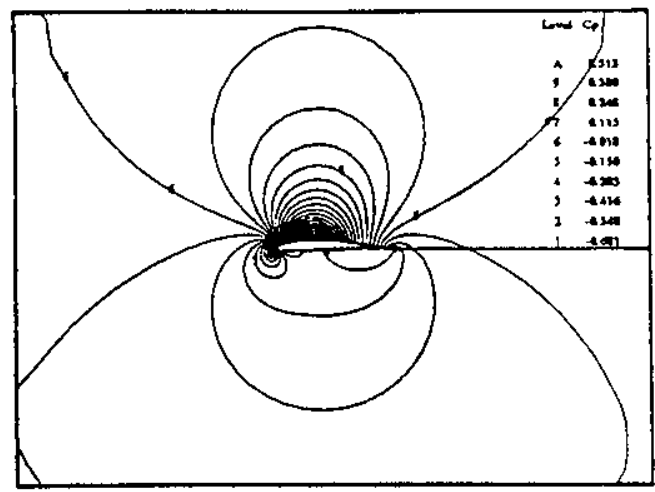

Figure 19. Pressure coefficient contours surrounding the Eppler 387 airfoil.

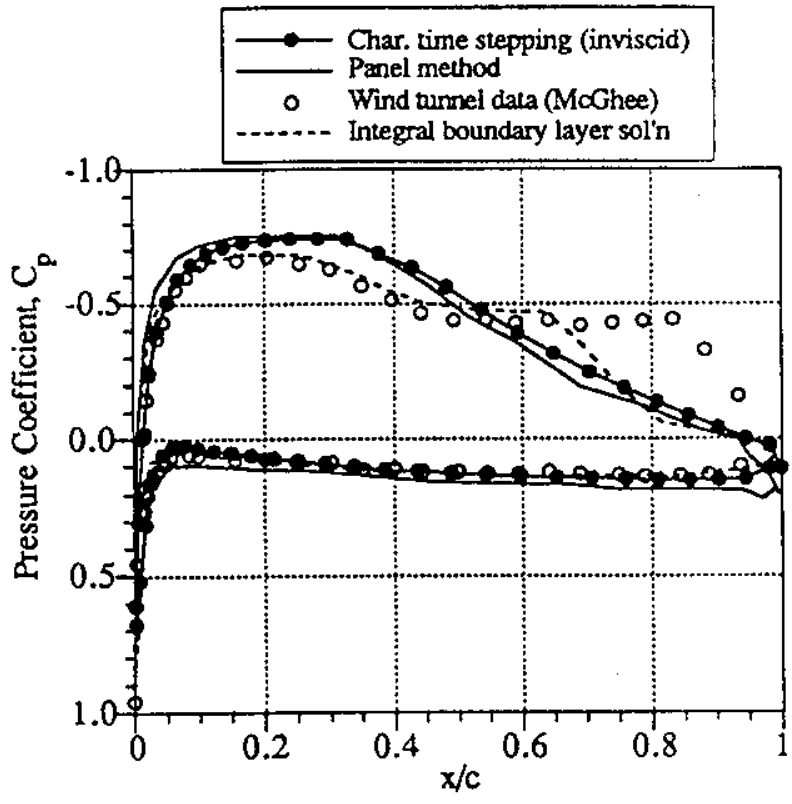

Figure 20. Pressure coefficient on the Eppler 387 airfoil surface using preconditioned Euler, experimental and potential methods.

the benefits of preconditioning decrease. Convergence rate is accelerated for all cases at the nearly sonic inflow condition.

\section{Summary}

The theoretical and actual convergence-rate acceleration for the above test cases is summarized in Table 2.

\section{Navier-Stokes Preconditioning}

The idea behind characteristic time stepping is to transform as closely as possible the behavior of a system of equations into that of a scalar equation. The

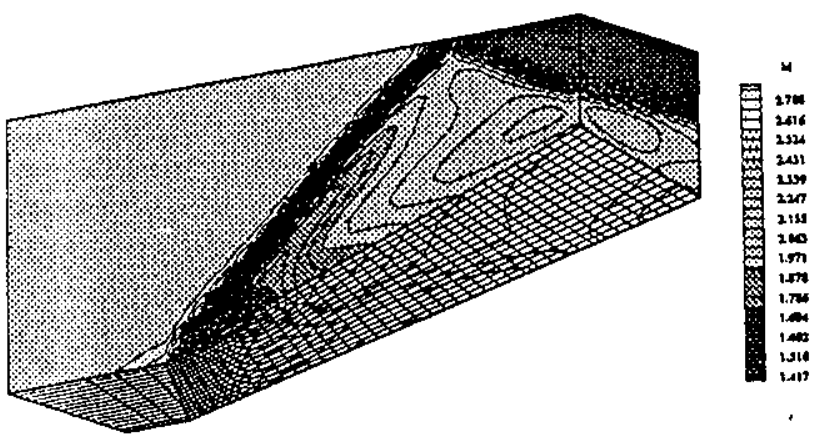

Figure 21. Flooded and line contours of pressure and a representative section of the mesh for the threedimensional wedge with inflow $M=2.8$. Flooded contours are shown on the left wall and the outflow plane. Line contours and the mesh rest upon the compression ramp.

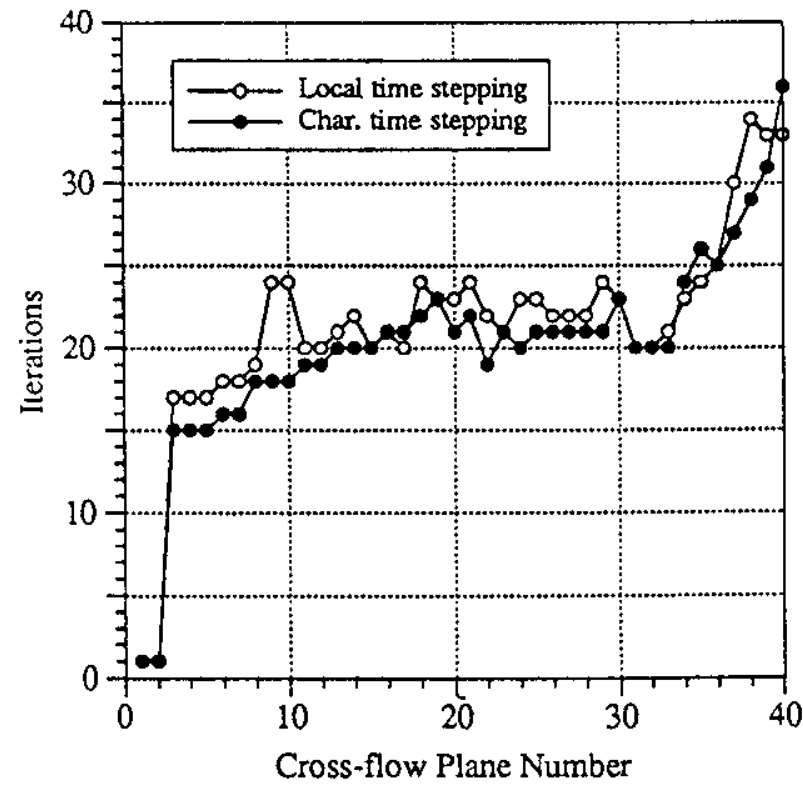

Figure 22. Iterations per plane for three-dimensional wedge with inflow Mach number of $M=2.8$ and approximate factorization in the cross flow plane.

progress of this transition can be visualized with the Fourier footprint. The Fourier footprint reflects the stability of a first-order ordinary differential equation and thus any positive real part causes disturbances to grow exponentially.

The footprint results from determining the eigenvalues of the Fourier transform of a discrete time step, $\Delta t$, times the semi-discrete form of the residual. For the first-order scalar wave equation, the Fourier footprint 


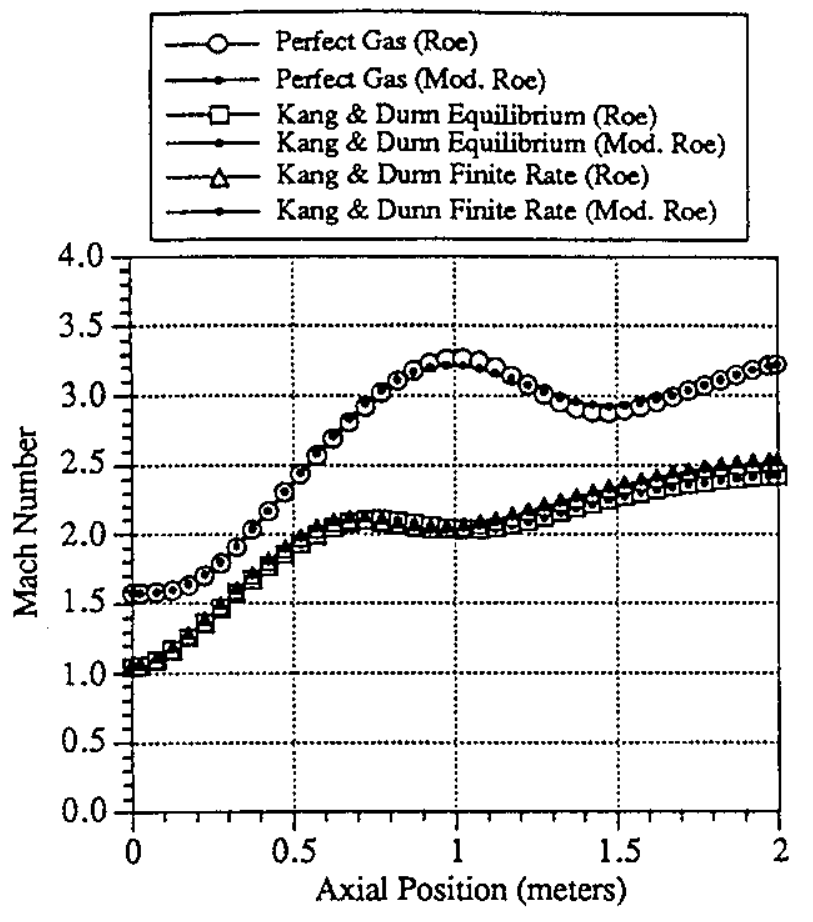

Figure 23. Streamwise Mach-number distribution on the centerline of the axi-symmetric nozzle.

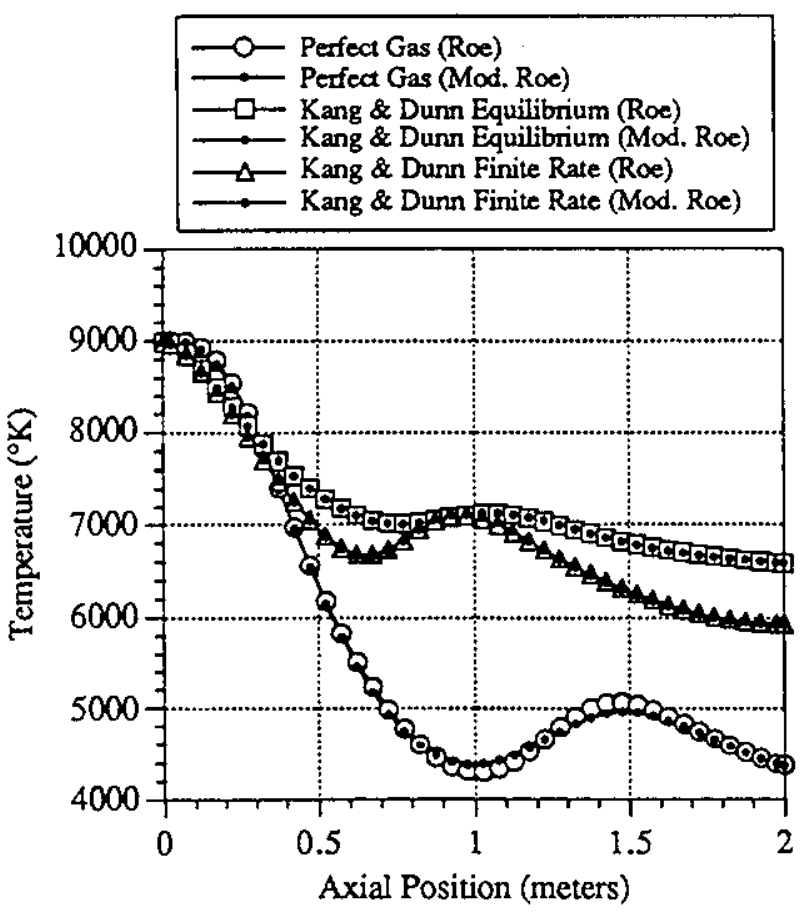

Figure 24. Streamwise temperature distribution on the centerline of the axi-symmetric nozzle.

is a circle with a Courant-number radius resting in the negative real half of the complex plane. The final criterion for numerical stability is set down by the method of time integration. The footprint and the characteris-

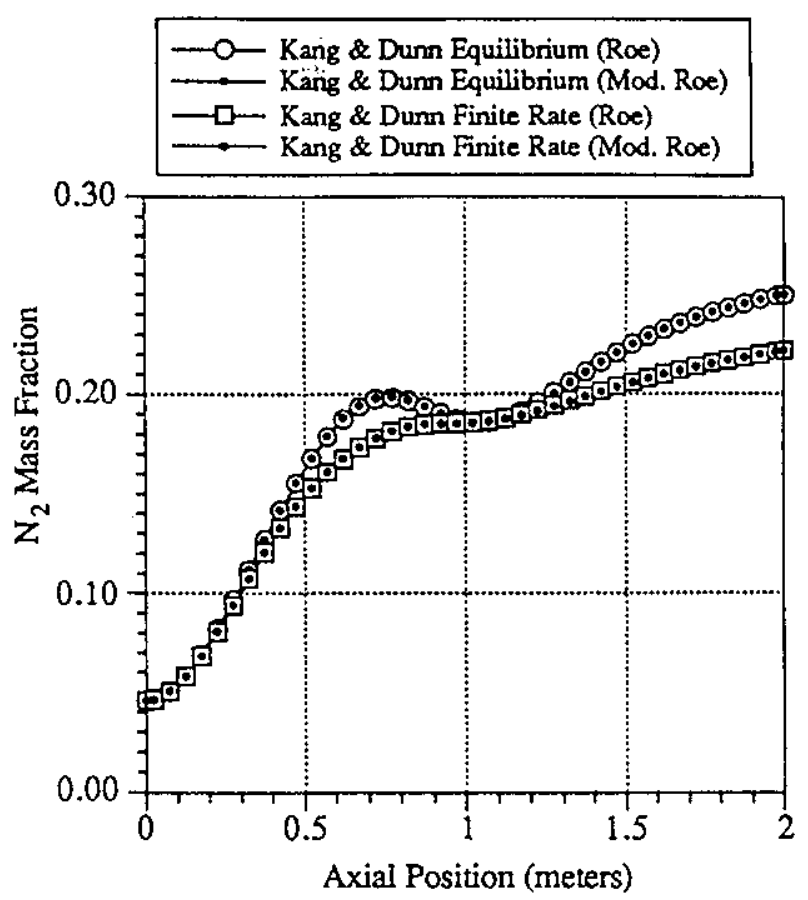

Figure 25. Streamwise diatomic ṇitrogen massfraction distribution on the centerline of the axi-symmetric nozzle.

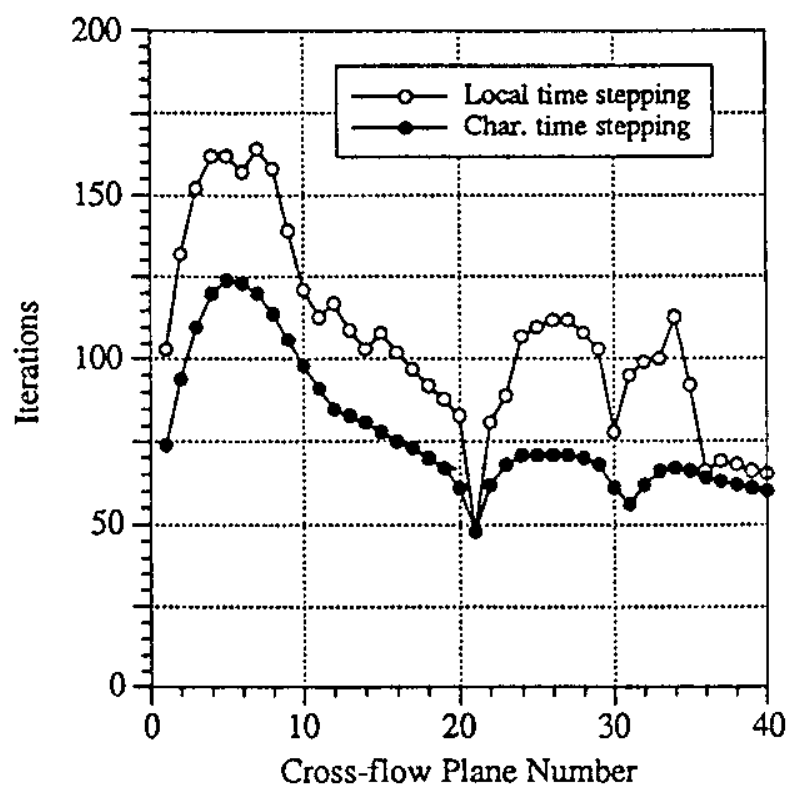

Figure 26. Iterations per plane for reacting-nozzle case assuming air as a perfect gas.

tic polynomial of the time evolution scheme determines which waves are most effectively damped [22].

The one-dimensional preconditioned Euler equations share the same Fourier footprint as the scalar wave equation. However, if we include the viscous 


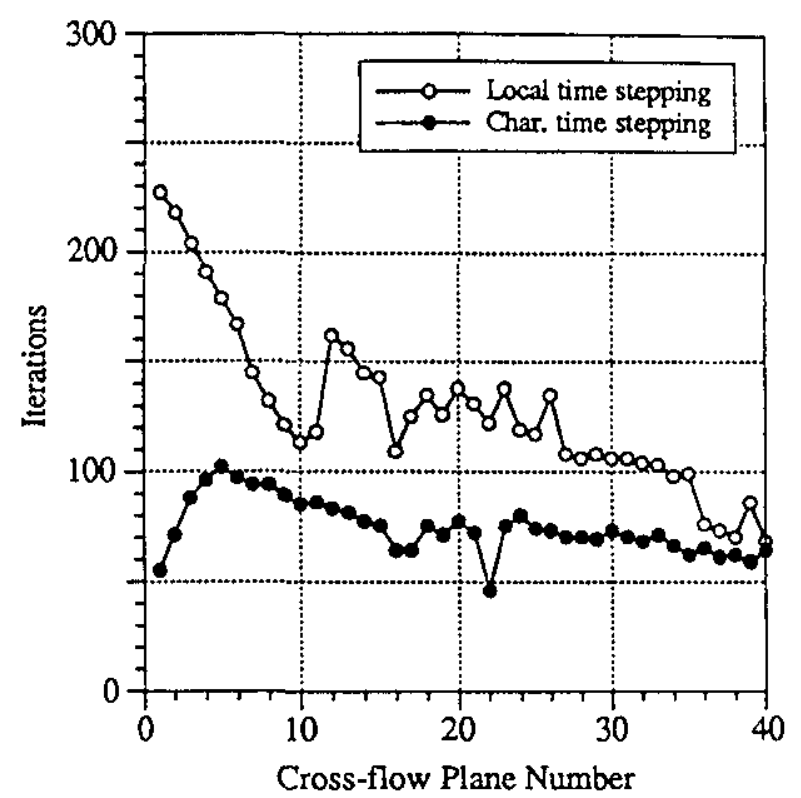

Figure 27. Iterations per plane for diverging nozzle with a real gas assuming five-species equilibrium flow.

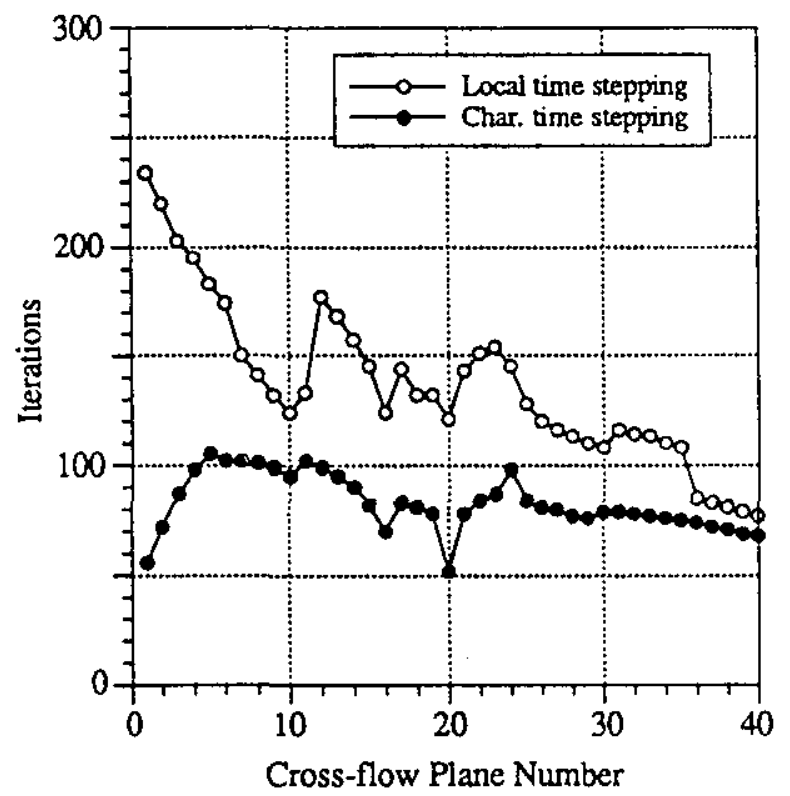

Figure 28. Iterations per plane for reacting-nozzle case assuming finite-rate reactions with three nonequilibrium vibration species.

terms which comprise the Navier-Stokes equations, the Fourier footprint becomes prohibitively skewed as the cell Reynolds number approaches zero, $R e_{\Delta x} \rightarrow 0$. This footprint is shown in Fig. 29. Note that the axes are not one-to-one and that the footprints for high Reynolds numbers are nearly circular. The conclusion is that the convective and acoustic waves in the low Reynolds num-

\begin{tabular}{|c|c|c|c|}
\hline Test Case & Mach No. & Theory & Actual \\
\hline V.L.S. Channel & 0.001 & 1001 & N/A \\
\hline X. Channel & 0.85 & 6.50 & 3.56 \\
\hline Eppler 387 & 0.05 & 21.0 & N/A \\
\hline 3-D Ramp & 2.80 & 1.97 & 0.92 .1 .33 \\
\hline Nozzle & 1.046 & 44.5 & 4.27 \\
\hline
\end{tabular}

Table 2. Theoretical and actual convergence-rate acceleration for the presented test cases. N/A indicates test cases that were impractical without preconditioning.

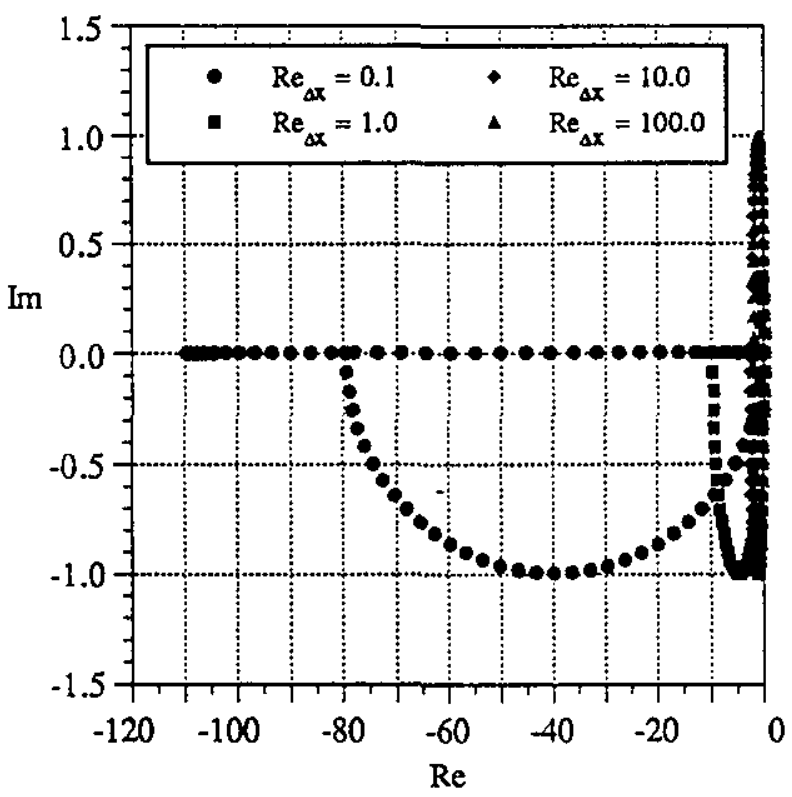

Figure 29. Fourier footprint using the inviscid preconditioning on the one-dimensional Navier-Stokes equations with unit CFL, $\nu=1$.

ber viscous limit cause more stiffness than the original equations.

What do we want the footprint to look like? The one-dimensional heat equation yields a footprint which lies on the negative real axis with no imaginary part. 
The footprint is bounded by zero and twice the Courant number. We would hope that as the cell Reynolds number approaches the viscous limit, the Fourier footprint would approach the footprint of the heat equation for all three waves in the system. To accomplish this, we must derive a new preconditioning matrix that is designed for the task.

To determine the viscous preconditioning matrix, we adopt the symmetrization variables proposed by Abarbanel, et.al. [29]. These variables symmetrize both the inviscid and viscous flux Jacobians, which is beneficial because we can then determine a positive definite preconditioner. These variables are also known in differential form as

$$
d Z \equiv\left\{\begin{array}{c}
\frac{a}{\sqrt{\gamma}} \frac{d \rho}{\rho} \\
\frac{a}{\sqrt{\gamma(\gamma-1)}} \frac{d T}{T}
\end{array}\right\} .
$$

The linearized Navier-Stokes equations are then

$$
\frac{\partial Z}{\partial t}=-\left(A \frac{\partial Z}{\partial x}+A_{v} \frac{\partial^{2} Z}{\partial x^{2}}\right)
$$

where

$$
A=\left[\begin{array}{ccc}
u & a \frac{1}{\sqrt{\gamma}} & 0 \\
a \frac{1}{\sqrt{\gamma}} & u & a \sqrt{\frac{\gamma-1}{\gamma}} \\
0 & a \sqrt{\frac{\gamma-1}{\gamma}} & u
\end{array}\right],
$$

and

$$
A_{v}=\left[\begin{array}{ccc}
0 & 0 & 0 \\
0 & \frac{4}{3} \nu & 0 \\
0 & 0 & \frac{\gamma}{P_{r}} \nu
\end{array}\right] .
$$

For convenience we define matrices, $\tilde{A}$ and $\tilde{A}_{v}$, which are non-dimensionalized by the velocity and kinematic viscosity, $u$ and $\nu$, respectively. These matrices are

$$
\tilde{A} \equiv \frac{1}{u} A=\left[\begin{array}{ccc}
1 & \frac{1}{M} \frac{1}{\sqrt{\gamma}} & 0 \\
\frac{1}{M} \frac{1}{\sqrt{\gamma}} & 1 & \frac{1}{M} \frac{\sqrt{\gamma-1}}{\sqrt{\gamma}} \\
0 & \frac{1}{M} \frac{\sqrt{\gamma-1}}{\sqrt{\gamma}} & 1
\end{array}\right],
$$

and

$$
\tilde{A}_{v} \equiv \frac{1}{\nu} A_{v}=\operatorname{diag}\{0,4 / 3, \gamma / \operatorname{Pr}\} .
$$

The Fourier footprint of the preconditioned NavierStokes equations with Roe's approximate Riemann solver and second order central differencing is

$$
\begin{aligned}
\mathcal{F}\left\{\Delta t P \frac{\partial Z}{\partial t}\right\}= & -i \nu_{x} P \vec{A} \sin \beta_{x} \\
& -2 \nu_{x} P|\tilde{A}| \sin ^{2}\left(\beta_{x} / 2\right) \\
& -\frac{4 \nu_{x}}{R e_{\Delta x}} P \tilde{A}_{v} \sin ^{2}\left(\beta_{x} / 2\right)
\end{aligned}
$$

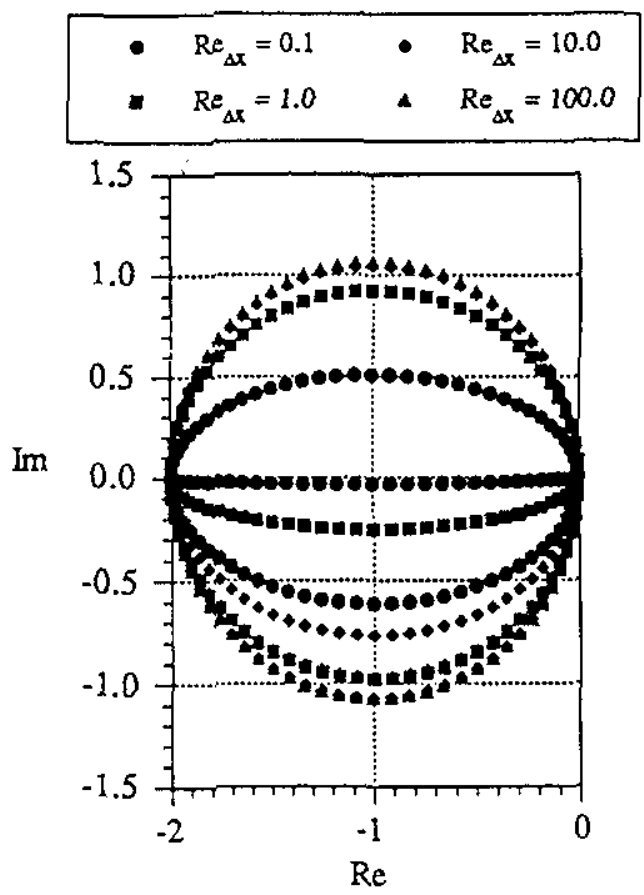

Figure 30. Fourier footprint using viscous preconditioning on the one-dimensional Navier-Stokes equations.

This can be rearranged by separating the real and imaginary parts to give

$$
\begin{aligned}
\mathcal{F}\left\{\Delta t P \frac{\partial Z}{\partial t}\right\}= & -2 \nu_{x} P\left(|\tilde{A}|+\frac{2 \tilde{A}_{v}}{R e_{\Delta x}}\right) \sin ^{2}\left(\beta_{x} / 2\right) \\
& -i \nu_{x} P \tilde{A} \sin \beta_{x} .
\end{aligned}
$$

To control the growing negative real part, we must choose the viscous preconditioning matrix to be

$$
P=\left(|\tilde{A}|+\frac{2 \tilde{A}_{v}}{R e_{\Delta x}}\right)^{-1} .
$$

With this formulation, we recover the inviscid preconditioner in the inviscid limit of $R e_{\Delta x} \rightarrow \infty$. The Fourier footprint for this preconditioning matrix is shown in Fig. 30 for several representative cell Reynolds numbers. Notice that as the cell Reynolds number becomes large, the footprint becomes circular. Also, notice the controlled behavior for decreasing cell Reynolds numbers. All three waves are slowly collapsing onto the negative real axis as totally dissipative waves. Finally, notice that the high-frequency acoustic and entropy waves are forced to meet at the real axis.

As an initial plausibility test case, we consider the simple damping of a $5 \%$ pressure disturbance in the center of a $M=0.1$ flow field. A pressure disturbance couples both the linear convective and the 


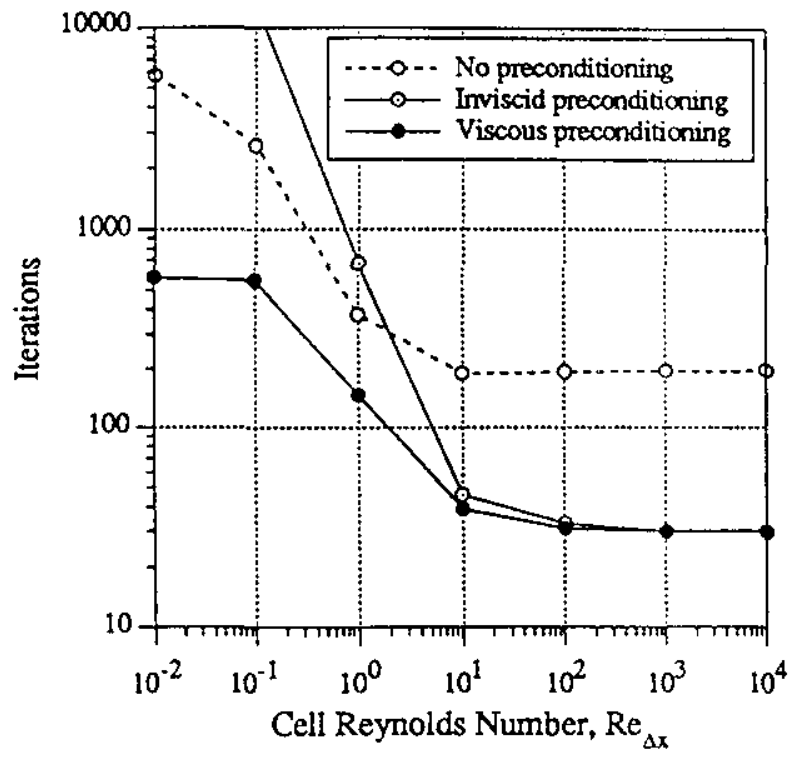

Figure 31. Iterations required to damp a $5 \%$ pressure disturbance in a $M=0.1$ free stream at various cell Reynolds numbers.

non-linear acoustic waves. We use MUSCL differencing with Roe's scheme and second-order central differencing for the viscous fluxes and investigate the convergence rate at several cell Reynolds numbers. Since Euler explicit time integration with $\kappa=1 / 3$ is unstable for some waves, we use the optimal 3-stage scheme of Van Leer, et.al. [22]. The recommended stage weights are $\alpha_{1}=0.2884, \alpha_{2}=0.5010$ and $\alpha_{3}=1.0$ with a Courant number of $\nu=1.3254$.

We compare the proposed viscous preconditioning with the inviscid preconditioning of Equ. (19) with $\tau=1$ and include the standard scheme without preconditioning. The results are shown in Fig. 31. In the inviscid limit the inviscid and viscous preconditioners converge the normalized residual to a tolerance of 10 orders in 30 iterations. Without preconditioning convergence is met in 195 iterations. At $M=0.1$, the expected convergence rate acceleration for the one-dimensional Euler equations is 11 .

Notice how the performance of the inviscid preconditioner deteriorates as the cell Reynolds number decreases. The threshold is around $R e_{\Delta x} \approx 2$. We have observed the same behavior for the two-dimensional preconditioning. The viscous preconditioning holds true to form for all cell Reynolds numbers and converges the lowest Reynolds number case in 572 iterations. This is compared to 5767 iterations without preconditioning. Running the inviscid preconditioning at the low end was impractical. We estimate convergence in more than 100,000 iterations for $R e_{\Delta x}=0.01$.

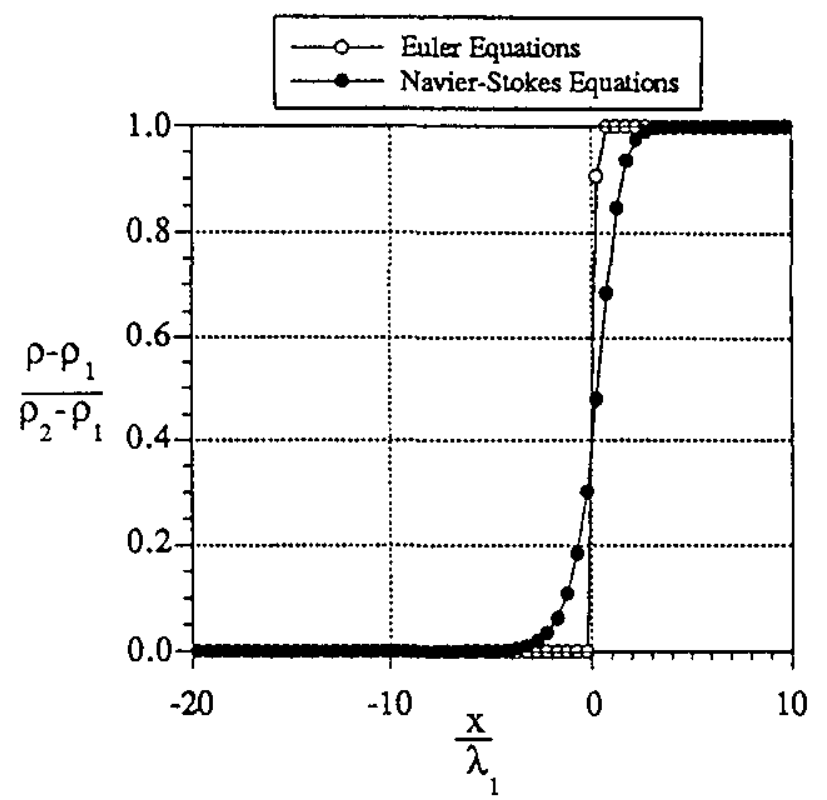

Figure 32. Internal normal shock structure. Shown is the inviscid and viscous normalized density distribution.

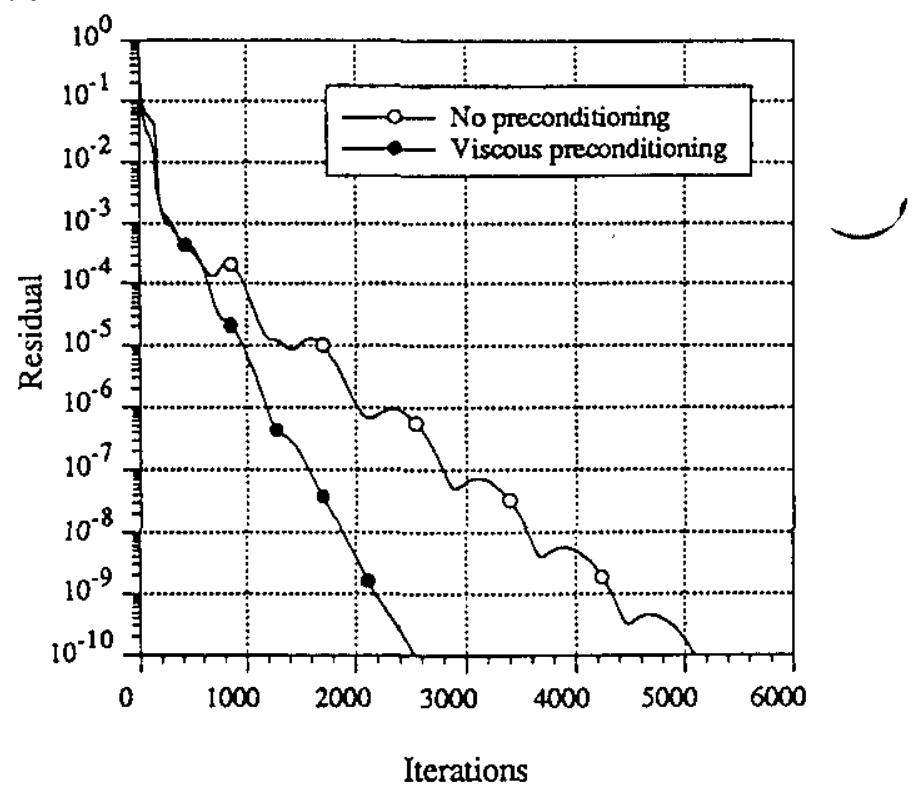

Figure 33. Residual histories for the computation of an internal normal shock structure.

Note that a Blasius profile simulation has cell Reynolds numbers on the order of those in this example.

To compute a more realistic problem, we consider the internal structure of a normal shock with upstream Mach number of $M=2.0$ and a cell Reynolds number of $R e_{\Delta x} \approx 1.5$. The domain is bounded by a distance of 45 mean free paths upstream and downstream of the shock. This problem was proposed by Eppard and Grossman [27] during their examination of real-gas ef- 
fects at hypersonic Mach numbers for monatomic and diatomic gases. Our normal shock solution is shown along with the inviscid solution in Fig. 32. The viscous test case did not require a limiter. Residual histories with and without viscous preconditioning are shown in Fig. 33. Convergence is obtained in 2536 and 5091 iterations, respectively.

\section{Conclusions}

The formulation for extending to finite-rate chemistry the matrix preconditioning of Van Leer is presented. Perfect-gas computations confirm convergence acceleration at all subsonic Mach numbers with significant improvement in the transonic and incompressible regimes. Inviscid incompressible flows are quickly and efficiently calculated using a compressible formulation and eigenvalue stiffness is effectively reduced. Results for marching a chemically reacting flow proves likewise beneficial at low supersonic speeds. Implementation is given for both explicit and implicit time integration. In addition, the one-dimensional Navier-Stokes equations are effectively preconditioned. Future work must robustly simulate all flow regimes, namely the realm of the complete Navier-Stokes equations.

\section{Acknowledgements}

This work was sponsored under NASA grant NAG1-776 and Air Force grant F49620-92-J-0158.

\section{References}

1. Van Leer, B., Lee, W.T., and Roe, P., "Characteristic Time-Stepping or Local Preconditioning of the Euler Equations," ALAA 91-1552, Honolulu, Hawaii, June, 1991.

2. Chorin, A.J., "A Numerical Method for Solving Incompressible Viscous Flow Problems," Journal of Computational Physics, 2, 1967.

3. Turkel, E., "Preconditioned Methods for Solving the Incompressible and Low Speed Compressible Equations," Journal of Computational Physics, 72, pp. 277-298, 1987.

4. Merkle, C.L. and Choi, Y.H., "Computation of Low-Speed Flow with Heat Addition," ALAA Journal, pp. 831-838, June, 1987.

5. Viviand, H., "Pseudo-unsteady Systems for Steady Inviscid Flow Calculations," Numerical Methods for the Euler Equations of Fluid Dynamics, 1985.
6. Grossman, B. and Cinnella, P., "Flux-Split Algorithms for Flows with Non-equilibrium Chemistry and Vibrational Relaxation," Journal of Computational Physics, 88, pp. 131-168, 1990.

7. Grossman, B. and Walters, R.W., "An Analysis of Flux-Split Algorithms for Euler's Equations with Real Gases," AIAA 87-1117-CP, 1987.

8. Vinokur, M. and Liu, Y., "Equilibrium Gas Flow Computations: II. An Analysis of Numerical Formulations of Conservation Laws," AIAA 88-0127, June, 1988.

9. Glaister, P., "An Approximate Linearised Riemann Solver for the Euler Equations for Real Gases," Journal of Computational Physics, 74, pp. $382-408,1988$.

10. Liou, M.-S., Van Leer, B., and Shuen, J.-S., "Inviscid Flux Splitting Algorithms for Real Gases with Nonequilibrium Chemistry," NASA TM-100856, 1988.

11. Walters, R.W., Cinnella, P., Slack, D.C., and Halt, D., "Characteristic-Based Algorithms for Flows in Thermo-Chemical Nonequilibrium," AIAA 900393, Reno, Nevada, January, 1990.

12. Walters, R.W., Cinnella, P., and Slack, D.C., "A Status Report on GASP - A General Aerodynamic Simulation Program," Seventh National Aero-Space Plane Symposium, Paper No. 9, NASA Lewis, 1989.

13. Withington, J.P., Shuen, J.S., and Yang, V., "A Time Accurate, Implicit Method for Chemically Reacting Flows at All Mach Numbers," AIAA 910581, Reno, Nevada, January, 1991.

14. Volpe, G., "On the Use and Accuracy of Compressible Flow Codes at Low Mach Numbers," ALAA 91-1662, Honolulu, Hawaii, June, 1991.

15. Rogers, S.E., Kwak, D., and Kiris, C., "Numerical Solution of the Incompressible Navier-Stokes Equations for Steady- State and Time-Dependent Problems," AIAA 89-0463, Reno, Nevada, January, 1989.

16. McGhee, R.J., Walker, B.S., and Millard, B.F., "Experimental Results for the Eppler 387 Airfoil at Low Reynolds Numbers in the Langley 
Low-Turbulence Pressure Tunnel," NASA TM4062,1988 .

17. Lee, W.T., "Local Preconditioning of the Euler Equations," Ph.D. Thesis, University of Michigan, 1991.

18. Thomas, J.L., Van Leer, B., and Walters, R.W., "Implicit Flux-Split Schemes for the Euler Equations," ALAA 85-1680, Cincinnati, Ohio, July, 1985.

19. Turkel, E., "Review of Preconditioning Methods for Fluid Dynamics," to appear in Applied Numerical Mathematics, 1992.

20. Rizzi, A., "Computation of Rotational Transonic Flow," Numerical Methods for the Computation of Inviscid Thansonic Flows with Shock Waves, A GAMM Workshop, p 153-166, 1981.

21. Rumsey, C.L., Van Leer, B., and Roe, P.L., "Effect of a Multi-dimensional Flux Function on the Monotonicity of Euler and Navier-Stokes Computations," AIAA 91-1530, 1991.

22. Van Leer, B., Tai, C.H., and Powell, K.G., "Design of Optimaly Smoothing Multi-Stage Schemes for the Euler Equations," ALAA 89-1933, 1989.

23. Dadone, A. and Grossman, B., "Surface Boundary Conditions for the Numerical Solution of the Euler Equations," Virginia Tech ICAM Report ICAM 92-10-04, 1992.

24. Choi, Y.H.. and Merkle, C.L., "The Application of Preconditioning in Viscous Flows," to appear in Journal of Computational Physics, 1992.

25. Choi, Y.H.. and Merkle, C.L., "Application of Time-Iterative Schemes to Incompressible Flows," ALAA Journal Vol. 23, no. 10, p 1518-1524, 1985.

26. Feng, J.. and Merkle, C.L., "Evaluation of Preconditioning Methods for Time-Marching Systems," AIAA 90-0016 Reno, Nevada, 1990.

27. Eppard, W.M. and Grossman, B., "Calculation of Hypersonic Shock Structure Using Flux-Split Algorithms," Virginia Tech ICAM Report ICAM 9107-04, 1991.

28. Kang, S.W., Dunn, M.G., and Jones, W.L., "Theoretical and Measured Electron-Density Distribu- tions for the RAM Vehicle at High Altitudes," ALAA 72-689, Boston, Massachusetts, June, 1972.

29. Abarbanel, S., Dutt, P., and Gottlieb, D., "Split ting Methods for Low Speed Mach Number Eule and Navier-Stokes Equations," NASA CR 178297 and ICASE Report No. 87-30, May, 1987. 


\section{Appendix}

\section{A. Preconditioning Matrix}

The preconditioning matrix for finite-rate chemistry in a stream-aligned coordinate system is

$$
P=\left[\begin{array}{cccccccccccc}
\frac{\tau}{\beta^{2}} M^{2} & 0 & \cdots & 0 & -\frac{\tau}{\beta^{2}} M & 0 & 0 & 0 & \cdots & \cdots & 0 & 0 \\
0 & 1 & & \vdots & 0 & \vdots & \vdots & \vdots & & & \vdots & \vdots \\
\vdots & & \ddots & 0 & \vdots & \vdots & \vdots & \vdots & & & \vdots & \vdots \\
0 & \cdots & 0 & 1 & 0 & 0 & 0 & 0 & \cdots & \cdots & 0 & 0 \\
-\frac{\tau}{\beta^{2}} M & 0 & \cdots & 0 & \frac{\tau}{\beta^{2}}+1 & 0 & 0 & 0 & \cdots & \cdots & 0 & 0 \\
0 & \cdots & \cdots & 0 & 0 & r & 0 & \vdots & & & \vdots & 0 \\
0 & \cdots & \cdots & 0 & 0 & 0 & \tau & 0 & \cdots & \cdots & 0 & 0 \\
0 & \cdots & \cdots & 0 & 0 & 0 & 0 & 1 & 0 & \cdots & 0 & 0 \\
\vdots & & & \vdots & \vdots & \vdots & \vdots & 0 & \ddots & & \vdots & \vdots \\
\vdots & & & \vdots & \vdots & \vdots & \vdots & \vdots & & \ddots & 0 & \vdots \\
0 & \cdots & \cdots & 0 & 0 & 0 & 0 & 0 & \cdots & 0 & 1 & 0 \\
0 & \cdots & \cdots & 0 & 0 & 0 & 0 & 0 & \cdots & \cdots & 0 & 1
\end{array}\right] .
$$

Here $\tau$ and $\beta$ are dependent upon the Mach number through

$$
\begin{aligned}
& \beta= \begin{cases}\sqrt{M^{2}-1}, & \text { if } M \geq 1 ; \\
\sqrt{1-M^{2}}, & \text { if } M<1,\end{cases} \\
& \tau= \begin{cases}\sqrt{1-M^{-2}}, & \text { if } M \geq 1 ; \\
\sqrt{1-M^{2}}, & \text { if } M<1 .\end{cases}
\end{aligned}
$$

\section{B. Explicit Preconditioning Matrix}

The preconditioning matrix, $\mathcal{P}$, for finite-rate chemistry in three dimensions is defined as

$$
\mathcal{P} \equiv \frac{\partial q}{\partial U} \frac{\partial U}{\partial \vec{U}} P \frac{\partial \vec{U}}{\partial U} \frac{\partial U}{\partial q}
$$

The matrix is

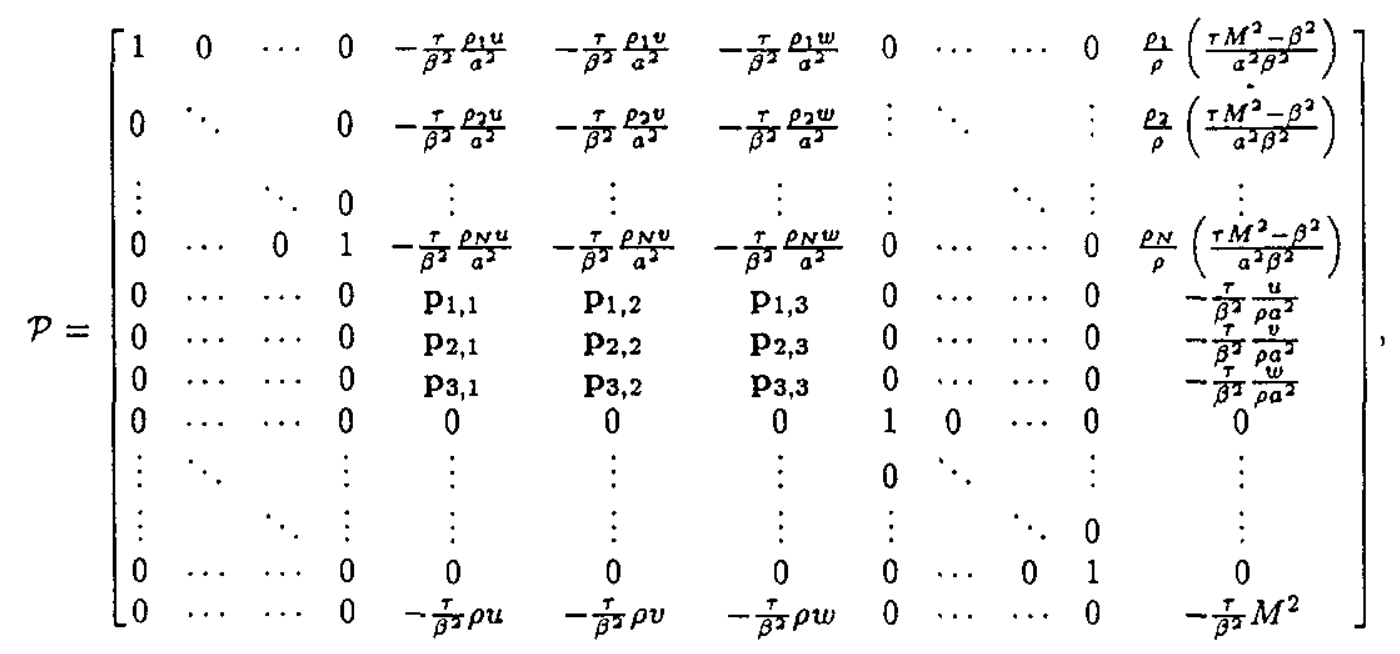

where

$$
\mathrm{p}=\frac{1}{q^{2}}\left[\left(1+\frac{\tau}{\beta^{2}}-\tau\right) \overrightarrow{\mathrm{V}}^{T} \overrightarrow{\mathrm{V}}+\tau q^{2} \mathbf{I}\right]
$$




\section{Impliclt Precondltoning Matrix} is

The inverse of the preconditioning matrix, $\mathcal{P}^{-1}$, is used in the implicit time integration implementation and

$$
\mathcal{P}^{-1}=\left[\begin{array}{cccccccccccc}
1 & 0 & \cdots & 0 & \rho_{1} \frac{u}{q^{2}} & \rho_{1} \frac{v}{q^{2}} & \rho_{1} \frac{w}{q^{2}} & 0 & \cdots & \cdots & 0 & \frac{\rho_{1}}{\rho q^{2}}\left(1-M^{2}+\frac{\beta^{2}}{\tau}\right) \\
0 & \ddots & & 0 & \rho_{2} \frac{u}{q^{2}} & \rho_{2} \frac{v}{q^{2}} & \rho_{2} \frac{w}{q^{2}} & \vdots & \ddots & & \vdots & \frac{\rho_{3}}{\rho q^{2}}\left(1-M^{2}+\frac{\beta^{2}}{\tau}\right) \\
\vdots & & \ddots & 0 & \vdots & \vdots & \vdots & \vdots & & \ddots & \vdots & \vdots \\
0 & \cdots & 0 & 1 & \rho_{N} \frac{u}{q^{2}} & \rho_{N} \frac{v}{q^{2}} & \rho_{N} \frac{w}{q^{2}} & 0 & \cdots & \cdots & 0 & \frac{\rho N}{\rho q^{2}}\left(1-M^{2}+\frac{\beta^{2}}{\tau}\right) \\
0 & \cdots & \cdots & 0 & \Pi_{1,1} & \Pi_{1,2} & \Pi_{1,3} & 0 & \cdots & \cdots & 0 & \frac{u}{\rho q^{2}} \\
0 & \cdots & \cdots & 0 & \Pi_{2,1} & \Pi_{2,2} & \Pi_{2,3} & 0 & \cdots & \cdots & 0 & \frac{\frac{\rho}{\rho q^{2}}}{\rho g^{2}} \\
0 & \cdots & \cdots & 0 & \Pi_{3,1} & \Pi_{3,2} & \Pi_{3,3} & 0 & \cdots & \cdots & 0 & 0 \\
0 & \cdots & \cdots & 0 & 0 & 0 & 0 & 1 & 0 & \cdots & 0 & \vdots \\
\vdots & \ddots & & \vdots & \vdots & \vdots & \vdots & 0 & \ddots & & \vdots & \vdots \\
\vdots & & \ddots & \vdots & \vdots & \vdots & \vdots & \vdots & & \ddots & 0 & \vdots \\
0 & \cdots & \cdots & 0 & 0 & 0 & 0 & 0 & \cdots & 0 & 1 & 0 \\
0 & \cdots & \cdots & 0 & \frac{\rho u}{M^{2}} & \frac{\rho v}{M^{2}} & \frac{\rho w}{M^{2}} & 0 & \cdots & \cdots & 0 & \frac{\tau+\beta^{2}}{\tau M^{2}}
\end{array}\right]
$$

where

$$
\Pi=\frac{1}{q^{2}}\left[\frac{(\tau-1)}{\tau} \overrightarrow{\mathbf{V}}^{T} \overrightarrow{\mathbf{V}}+\frac{q^{2}}{\tau} \mathbf{I}\right]
$$

\section{Modified Fiux Function}

The modified Roe scheme for three-dimensional implementation with finite-rate chemistry may be written

$$
\hat{f}_{j+1 / 2}=\frac{1}{2}\left(\hat{f}_{j}+\hat{f}_{j+1}\right)-\frac{1}{2} \sum_{i=1}^{3}[f]_{i}
$$

where

$$
\begin{aligned}
& {[f]_{1}=\frac{-[\rho]}{\tilde{a}^{2}}|\tilde{\tilde{u}}|\left\{\begin{array}{c}
\tilde{\rho}_{1} / \tilde{\rho} \\
\vdots \\
\tilde{\rho}_{N} / \tilde{\rho} \\
\tilde{u} \\
\tilde{v} \\
\tilde{w} \\
\rho_{1} e_{n_{1}} / \tilde{\rho} \\
\vdots \\
\frac{\rho_{M} e_{n_{M}} / \tilde{\rho}}{\Theta_{1}}
\end{array}\right\}+|\tilde{\tilde{u}}|\left\{\begin{array}{c}
{\left[\rho_{1}\right]} \\
\vdots \\
\tilde{u}[\rho]+\tilde{\rho}[u] \\
\tilde{v}[\rho]+\tilde{\rho}[v] \\
\tilde{w}[\rho]+\tilde{\rho}[w] \\
{\left[\rho_{1} e_{n_{1}}\right]} \\
\vdots \\
{\left[\rho_{M} e_{n_{M}}\right]} \\
\Theta_{2}
\end{array}\right\}}
\end{aligned}
$$

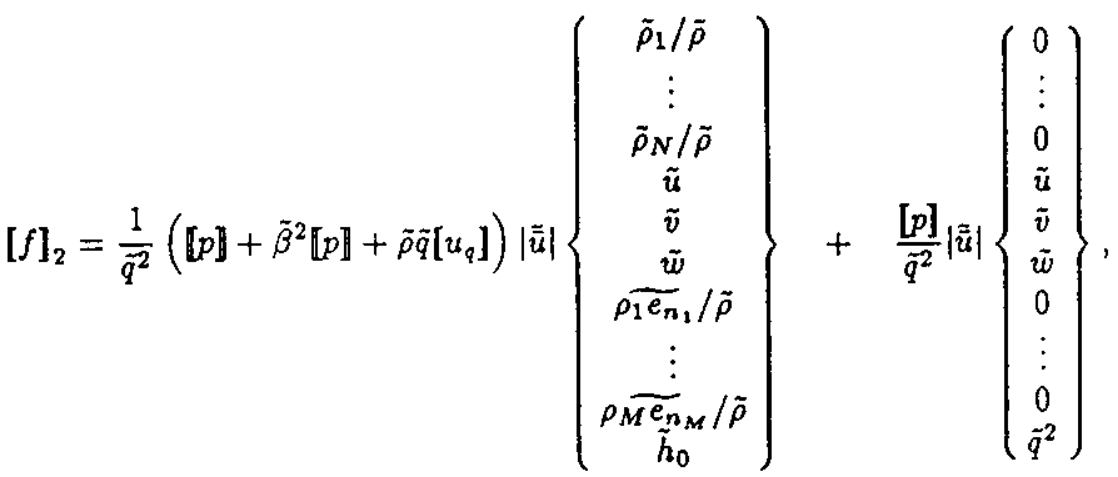




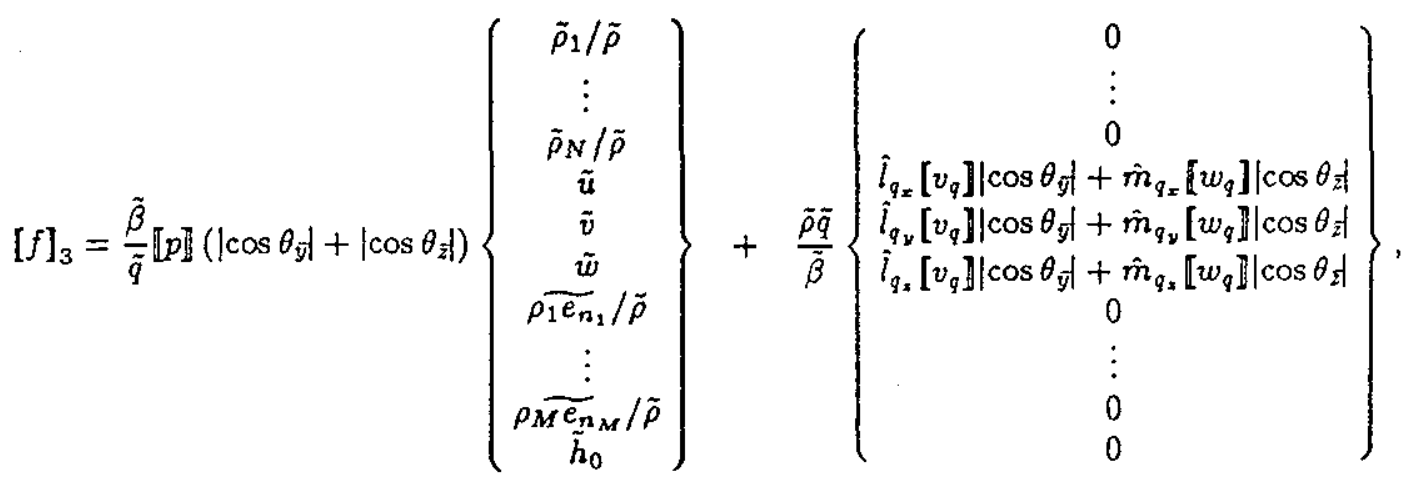

where

$$
\begin{gathered}
\Theta_{1} \equiv \tilde{h}_{0}-\frac{\tilde{a}^{2}}{\tilde{\tilde{\gamma}}-1} \\
\Theta_{2} \equiv \sum_{i=1}^{N} \tilde{\beta}_{i}\left[\rho_{i} \rrbracket+\sum_{j=1}^{M}\left[\rho_{j} e_{n_{j}}\right]+\tilde{\rho} \tilde{u}[u]+\tilde{\rho} \tilde{v}[v]+\tilde{\rho} \tilde{w}[w] .\right.
\end{gathered}
$$

Two sets of direction cosines are involved in the modified Roe algorithm. One set, $(\cdot)_{q_{x}},(\cdot)_{q_{y}},(\cdot)_{q_{s}}$, transforms from Cartesian coordinates to the Roe average stream-aligned coordinate system. This transformation may be determined by many different axis rotation sequences and is therefore somewhat arbitrary. The velocity vector in Cartesian coordinates has components $\{\tilde{u}, \tilde{v}, \tilde{w}\}$, so the unit vector along the stream-aligned coordinate is

$$
\vec{k}_{q} \equiv\{\tilde{u} / \tilde{q}, \tilde{v} / \tilde{q}, \tilde{w} / \tilde{q}\} .
$$

The transformation used here is

$$
\left[\begin{array}{lll}
\hat{k}_{q_{x}} & \hat{k}_{q_{y}} & \hat{k}_{q_{x}} \\
\hat{l}_{q_{x}} & \hat{l}_{q_{y}} & \hat{l}_{q_{x}} \\
\hat{m}_{q_{x}} & \hat{m}_{q_{y}} & \hat{m}_{q_{x}}
\end{array}\right]=\left[\begin{array}{ccc}
\cos \phi & \cos \theta \sin \phi & \sin \theta \sin \phi \\
-\sin \phi & \cos \theta \cos \phi & \sin \theta \cos \phi \\
0 & -\sin \theta & \cos \theta
\end{array}\right] .
$$

The angles $\phi, \theta$ are determined from the velocity components as $\tan \theta=w / v$ and $\tan \phi=\sqrt{v^{2}+w^{2}} / u$. Note especially that a singularity occurs for $u=q$, (i.e. the flow is perfectly aligned with the Cartesian $x$-axis). This case represents the only flow condition that does not require a similarity transformation, or, equivalently, the similarity transformation should be the identity matrix. To accommodate all flow cases a small perturbation on the order of the machine's accuracy should be added to the $y$ component of the velocity. With this change, $\cos \theta=1$ and $\sin \theta=0$ for the case when $u=q$, and the identity matrix is the similarity transformation. The jumps in contravariant velocity are constructed as

$$
\begin{aligned}
{\left[u_{q}\right\rceil } & \left.\equiv \hat{k}_{q_{x}}[u]+\hat{k}_{q_{y}} \llbracket v\right]+\hat{k}_{q_{x}}[w] \\
\left.\llbracket v_{q}\right\rfloor & \equiv \hat{l}_{q_{x}}[u]+\hat{l}_{q_{y}}[v]+\hat{l}_{q_{s}}[w] \\
{\left[w_{q}\right\rceil } & \equiv \hat{m}_{q_{x}}[u]+\hat{m}_{q_{y}}[v]+\hat{m}_{q_{x}}[w] .
\end{aligned}
$$

The other set are the afore-mentioned direction cosines between the stream-aligned coordinate unit vectors and the cell face normal, $\vec{n}$. These are obtained with the dot product as

$$
\begin{aligned}
& \cos \theta_{\bar{x}} \equiv \vec{k}_{q} \cdot \vec{n} \\
& \cos \theta_{\bar{y}} \equiv \vec{l}_{q} \cdot \vec{n} \\
& \cos \theta_{\bar{z}} \equiv \vec{m}_{q} \cdot \vec{n} .
\end{aligned}
$$

The flow variables $\tilde{\rho}, \tilde{u}, \tilde{v}, \tilde{w}, \tilde{\rho}_{i}, \widetilde{\rho_{i} e_{n_{i}}}, \tilde{h}_{0}, \tilde{\beta}_{i}, \widetilde{\tilde{e}_{i}}, \tilde{\tilde{R}}, \bar{T}, \widetilde{\tilde{\gamma}}, c_{v}^{*}, \tilde{c}_{v}^{*}$, and $\tilde{a}^{2}$ are determined from Roe averages as follows.

$$
\tilde{\rho}=\sqrt{\rho_{R} \rho_{L}}
$$




$$
\begin{aligned}
& \tilde{u}=\frac{\langle u \sqrt{\rho}\rangle}{\langle\sqrt{\rho}\rangle}, \quad \tilde{v}=\frac{\langle v \sqrt{\rho}\rangle}{\langle\sqrt{\rho}\rangle}, \quad \tilde{w}=\frac{\langle w \sqrt{\rho}\rangle}{\langle\sqrt{\rho}\rangle}, \\
& \tilde{\rho}_{i}=\frac{\sqrt{\rho_{R}} \rho_{i_{L}}+\sqrt{\rho_{L}} \rho_{i_{R}}}{\sqrt{\rho_{R}}+\sqrt{\rho_{L}}}, \quad i=1, \ldots, N \\
& \widetilde{\rho_{j} e_{n_{j}}}=\frac{\sqrt{\rho_{R}}\left(\rho_{j} e_{n_{j}}\right)_{L}+\sqrt{\rho_{L}}\left(\rho_{j} e_{n_{j}}\right)_{R}}{\sqrt{\rho_{R}}+\sqrt{\rho_{L}}}, \quad j=1, \ldots, M \\
& \tilde{h}_{0}=\frac{\left\langle h_{0} \sqrt{\rho}\right\rangle}{\langle\sqrt{\rho}\rangle}, \quad \tilde{\beta}_{i}=\tilde{\tilde{e}_{i}}-\frac{R_{i} \tilde{T}}{\tilde{\tilde{\gamma}}-1}+\frac{\tilde{q}^{2}}{2}, \\
& \tilde{\tilde{e}}_{i}=\frac{\left\langle\tilde{e}_{i} \sqrt{\rho}\right\rangle}{\langle\sqrt{\rho}\rangle}, \quad \tilde{\tilde{R}}=\frac{\langle\tilde{R} \sqrt{\rho}\rangle}{\langle\sqrt{\rho}\rangle}, \quad \tilde{T}=\frac{\langle T \sqrt{\rho}\rangle}{\langle\sqrt{\rho}\rangle}, \\
& \tilde{\tilde{\gamma}}=1+\frac{\tilde{\tilde{R}}}{\tilde{c}_{v}^{*}} \\
& c_{v_{i}}^{*}=\frac{1}{\llbracket T \rrbracket} \int_{T_{\ell}}^{T_{R}} c_{v_{i}}(\tau) d \tau, \quad \vec{c}_{v}=\sum_{i=1}^{N} \frac{\tilde{\rho}_{i} c_{v_{i}}^{*}}{\tilde{\rho}} \\
& \tilde{a}^{2}=(\tilde{\tilde{\gamma}}-1)\left(\tilde{h}_{0}-\frac{\tilde{q}^{2}}{2}+\tilde{c}_{v}^{*} \tilde{T}-\sum_{i=1}^{N} \frac{\bar{\rho}_{i} \tilde{\tilde{e}_{i}}}{\tilde{\rho}}-\sum_{j=1}^{M} \frac{\widetilde{\rho_{j} \tilde{e}_{n_{j}}}}{\tilde{\tilde{\rho}}}\right) .
\end{aligned}
$$

The contravariant velocity is

$$
\tilde{\bar{u}} \equiv \hat{k}_{x} \ddot{u}+\hat{k}_{y} \tilde{v}+\hat{k}_{z} \tilde{w} .
$$

